\title{
A discrete MMAP for analysing the behaviour of a multi-state complex dynamic system subject to multiple events
}

- Juan Eloy Ruiz-Castro, M. Dawabsha

- A discrete MMAP for analysing the behaviour of a multi-state complex dynamic system subject to multiple events

- Discrete Event Dynamic Systems (2019) 29:1-29

- DOI: https://doi.org/10.1007/s10626-018-0274-0

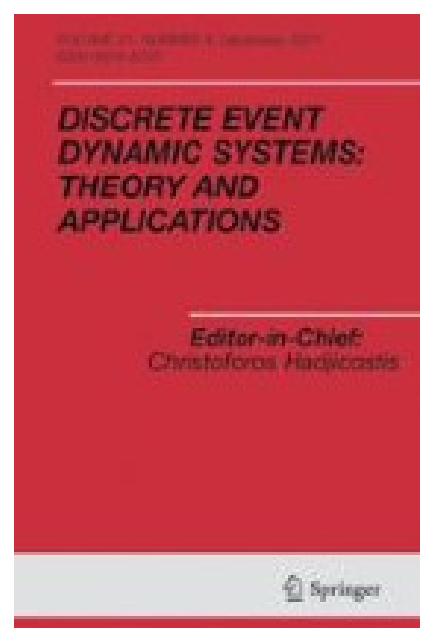




\title{
A discrete MMAP for analysing the behaviour of a multi-state complex dynamic system subject to multiple events
}

\author{
Juan Eloy Ruiz-Castro ${ }^{1}$ (D) M. Dawabsha ${ }^{1}$ \\ Received: 2 July 2017 / Accepted: 6 November 2018 / Published online: 8 December 2018 \\ (C) 2018 Springer Science+Business Media, LLC, part of Springer Nature
}

\begin{abstract}
A complex multi-state system subject to different types of failures, repairable and/or nonrepairable, external shocks and preventive maintenance is modelled by considering a discrete Markovian arrival process with marked arrivals (D-MMAP). The internal performance of the system is composed of several degradation states partitioned into minor and major damage states according to the risk of failure. Random external events can produce failures throughout the system. If an external shock occurs, there may be an aggravation of the internal degradation, cumulative external damage or extreme external failure. The internal performance and the cumulative external damage are observed by random inspection. If major degradation is observed, the unit goes to the repair facility for preventive maintenance. If a repairable failure occurs then the system goes to corrective repair with different time distributions depending on the failure state. Time distributions for corrective repair and preventive maintenance depend on the failure state. Rewards and costs depending on the state at which the device failed or was inspected are introduced. The system is modelled and several measures of interest are built into transient and stationary regimes. A preventive maintenance policy is shown to determine the effectiveness of preventive maintenance and the optimum state of internal and cumulative external damage at which preventive maintenance should be taken into account. A numerical example is presented, revealing the efficacy of the model. Correlations between the numbers of different events over time and in non-overlapping intervals are calculated. The results are expressed in algorithmic-matrix form and are implemented computationally with Matlab.
\end{abstract}

Keywords Reliability $\cdot$ Complex multi-state systems $\cdot$ Phase-type distribution $\cdot$ Marked Markovian Arrival Process (MMAP) · Correlations

Juan Eloy Ruiz-Castro jeloy@ugr.es

M. Dawabsha

dawabsha@correo.ugr.es

1 Department of Statistics and Operational Research and IEMath-GR, Faculty of Science, University of Granada, Campus Fuentenueva s/n., Granada, Spain 
Mathematics subject classification $60 \mathrm{~J} 10 \cdot 90 \mathrm{~B} 25 \cdot 60 \mathrm{~K} 10$

\section{Introduction}

A multi-state system is one that has a finite number of performance levels and various failure modes with different effects on the entire system performance. Examples of such a system include power supply systems and computer systems. The most elemental type is a binary system. However, when multiple states interact within a system, problems of a complex mathematical nature may arise. Since these systems were first addressed, by (Murchland 1975), multi-state reliability systems have been developed and applied intensively. In recent years, (Lisnianski et al. 2010) performed a comprehensive analysis of multi-state systems, and (Eryilmaz 2010) studied measures for single-unit multi-state systems and multi-state $k$-out-of$n$ : G systems. In this field, too, (Ruiz-Castro 2016a) analysed a complex multi-state system by considering Markov counting and reward processes.

Serious economic and human damage can be provoked when poor system reliability causes an unscheduled interruption or system failure. Preventive maintenance, which is employed to avoid this outcome, or at least to improve system reliability, involves regular, routine maintenance to help keep equipment up and running. Preventive maintenance has been discussed by (Osaki and Asakura 1970), who studied the behaviour of a two-unit system. (Mahfoud et al. 2016) reviewed this question and conducted a careful examination of the status of applicationoriented research into the preventive maintenance and optimisation of medical devices. In the field of survival analysis, (Yin et al. 2015) introduced models of condition-based maintenance (CBM), and (Laggounea et al. 2010) developed a preventive maintenance model to coordinate component replacements in a multi-component system.

A multi-state system can be analysed by various approaches. One class of stochastic models, which allows us to describe the behaviour of a complex multi-state system with multiple events and with correlated event times, is the Markov process with marked transitions. This class of counting processes makes it possible to model complex systems with wellstructured results, thanks to their matrix-algebraic form. Many reliability systems have inputs to the system over time, such as a repairable failure, a non-repairable failure, preventive maintenance or an external shock. When a multi-state system is considered, the number of events over time can be modelled through a Markovian arrival process (MAP). One class of distributions that is strongly related with MAPs is the phase-type distribution class (Neuts 1975; Neuts 1981). MAPs were also considered by (Neuts 1979), who observed that an interesting property of MAPs is that any stochastic counting process can be closely approximated by a sequence of MAPs. (He 2014) developed the theory of MAPs in an intuitive way, observing that MAPs with marked arrivals, or MMAPs, are an extension of MAPs when marked arrivals occur. An MMAP enables us to model complex multi-state systems in a wellstructured way, and to obtain results in an algorithmic and computational form. A warm standby system, considering a MMAP, was recently analysed by (Ruiz-Castro 2016b). A disadvantage of using MMAPs is the parameterisation effort required. This problem was analysed by (Buchholz et al. 2014), who made several proposals on how to estimate the parameters encountered in real problems.

Reliability systems are usually studied in the continuous case; nevertheless, not all systems can be continuously monitored, due to the internal structure of the system, the need for periodic inspections, etc., and instead are observed at certain epochs. A system can also be 
subject to periodic inspections, with the state of the system being identified at discrete times. Discrete reliability systems have been considered in electronic and aeronautical engineering (Warrington and Jones 2003). Moreover, discrete-time modelling has been used in studies of the fault debugging environment in the field of software reliability engineering. A discretetime model suitable for a periodic debugging schedule, describing maximum likelihood estimation for the model parameters, was presented by (Dewanji et al. 2011). This model can be used to estimate software reliability and has been applied to analyse such a problem in a unit of flight control software developed by the Indian Space Research Organization (ISRO). A new insight into the development of discrete-time modelling in software reliability engineering was given by (Shatnawi 2016). The Markovian structure has also been considered to study the evolution of reliability systems in discrete times. A complex $k$-out-of- $n: G$ system that evolved in discrete time through a discrete Markov process was modelled by (Ruiz-Castro and Li 2011).

\subsection{Motivation and contribution}

In reality, systems may fail in different ways. For example, a car tyre tread may fail due to wear or due to a puncture. In this example, there are two kinds of failure mode: soft failures caused by natural degradation and hard failures caused by external shock. A soft failure occurs as a result of natural deterioration, while a hard failure is the outcome of a destructive shock. A shock will not necessarily cause immediate system breakdown, but a negative impact will be provoked. Preventive maintenance is adopted to protect the system from hard failures and to maintain its functionality. Many models have been proposed to evaluate the reliability of faulttolerant systems subject to external shocks and internal degradation. For example, a generalised reliability system subject to degradation processes and to cumulative damage from external shocks was developed by (Li and Pham 2005). In a related field, (Liu et al. 2016) analysed the reliability of memory chips subject to a single-event upset and to a total ionising dose effect.

The model we present can be applied in fields such as civil, industrial and computer engineering. For instance, in computer engineering, the hard drive attached to a computer server is periodically inspected by an installed monitoring program that analyses logic and physics parameters to detect possible errors caused by internal and external events. In industrial engineering, any facility that requires a reliable electrical supply must have available generating sets capable of generating electricity in case of need. A genset is a diesel motor with a generator subject to repairable or total failures, for which preventive maintenance is necessary.

An interesting situation that can arise regarding preventive maintenance in the context of complex systems in which different types of failure may occur is when inspection reveals major damage to the system, which must then go to the repair facility for preventive maintenance, where different cost and time distributions may be present. We analyse this question by considering a maintenance policy from the standpoints of cost and reliability.

The present paper focuses on modelling a complex multi-state system that evolves in discrete time through a Markovian arrival process with marked arrivals (MMAP). This system is subject to several types of failure, repairable and/or non-repairable, as a consequence of internal wear or external shocks. Random events occur over time and if they impact on the system, diverse consequences can occur, including deterioration of internal performance, extreme failure or cumulative external damage. The internal performance state and that of 
cumulative external damage are partitioned according to the risk of failure: minor or major. Preventive maintenance is introduced, in conjunction with random inspection. If major internal or external damage is observed, the unit is sent to the repair facility for preventive maintenance. If a repairable failure occurs the unit is sent to the repair facility for corrective repair. The corrective repair and the preventive maintenance times have different distributions depending on the system state at which it failed or was observed. When a non-repairable failure occurs the device is replaced by an identical one. In this study, measures such as reliability, availability and expected number of events over time are obtained, and the correlation coefficient between two different types of events is determined and applied. Rewards and costs depending on the system state at which it failed or was inspected are included in the model. The model determines when preventive maintenance should be applied to optimise the behaviour of the system, from different standpoints. The modelling and the results obtained are presented in a matrix-algorithmic computational form, and are implemented computationally with Matlab.

This study extends previous research in this area in the following ways:

- The system passes through an indeterminate level of degradation, associated with its performance status. The unit is subject to failures that may be repairable or non-repairable, internal or accidental external.

- External shocks are incorporated in the model and can produce multiple consequences, such as extreme failure, cumulative external damage (a non-repairable failure if a threshold is reached) and aggravation of internal degradation or internal failure.

- Preventive maintenance is performed in response to random inspections.

- The major and minor states for internal performance and cumulative external damage can vary in number. The preventive maintenance policy will determine the most beneficial structure.

- The repair time distribution depends on the internal degradation status of the system.

- The preventive maintenance time distribution depends on the internal degradation and external cumulative damage observed by inspection.

- All results are expressed in algorithmic form, with PH distributions and Markovian Arrival Processes, with marked arrivals in discrete time (D-MMAP).

- Transient and long-term algorithmic analyses are performed. The stationary distribution is constructed using matrix analytic methods.

- Rewards and costs of repair and preventive maintenance, depending on the system state at which the unit failed or was inspected, are included.

- In addition a maintenance policy has been shown, we have calculated the optimum internal status and the optimum level of external cumulative damage when preventive maintenance should be carried out.

This paper is organised as follows. The system and its modelling are described in Section 2. The MMAP that governs the system is given in Section 3. The following section presents the transient and stationary distributions in an algorithmic form. Measures such as reliability, availability and the analysis of the time between events are addressed in Section 5. Section 6 then focuses on the mean number of events and correlations. Rewards are considered in Section 7, after which in Section 8 we show a maintenance policy. A numerical example to illustrate the versatility of the model is given in Section 9. Finally, the main conclusions drawn are summarised in Section 10. 


\section{The system and the model}

In this section, the assumptions underlying the system are described in detail. To model the system, the state-space must be well structured and so the system behaviour is modelled in a matrix-algorithmic form.

\subsection{Assumptions of the system}

We assume a multi-state complex system subject to repairable and/or non-repairable internal failures, external shocks and inspections. The internal performance of the system is composed of several states which are partitioned into two well-differentiated groups: minor and major damage states, which reflect a low and high risk of failure, respectively. From each of these operational states a repairable or non-repairable failure may occur. The unit is also exposed to external shocks. When a shock occurs and the system is operational, it may undergo one of three possible consequences: internal deterioration, cumulative external damage or extreme failure. Each time an external shock takes place, the cumulative external damage increases by passing through an external damage state. When the cumulative external damage reaches a given threshold, the unit undergoes a nonrepairable failure. In addition, when the unit undergoes an internal repairable failure, the system is sent to the repair facility for corrective repair. Analogously to the internal case, the cumulative external damage states are partitioned into minor and major damage states. Finally, an external shock may produce an extreme non-repairable failure. After a non-repairable failure, whether internal or the consequence of an external shock, the unit is removed and replaced by an identical one. Preventive maintenance is introduced into the system in response to random inspections, of which periodic inspection is a particular case. When an inspection takes place, the internal and the cumulative external damage states are observed. If a major internal or cumulative external damage state is observed, the unit is sent to the repair facility for preventive maintenance. The time distributions for repairs and preventive maintenance depend on the system state when inspection was performed. The repair facility is staffed by one repairperson. The system is based on the following assumptions.

Assumption 1. The internal operational time of the unit system is $P H$-distributed with representation $(\alpha, \mathbf{T})$ with order $n$. The $n$ operational states are partitioned into minor damage states (the first $n_{1}$ states) and major damage states (states $n_{1}+1, \ldots, n$ ). State 1 indicates that the system does not present significant damage.

Assumption 2. When an internal failure occurs, it may be repairable or non-repairable. The probability of the system undergoing a repairable or non-repairable failure from a transient state is given by the column vectors $\boldsymbol{T}_{r}^{0}$ and $\boldsymbol{T}_{n r}^{0}$, respectively. The probability of failure from the internal state $i$ at one step is given by the $i$-th element of the column vector $\boldsymbol{T}^{0}=\boldsymbol{T}_{r}^{0}+\boldsymbol{T}_{n r}^{0}$.

Assumption 3. Events that may produce failures of the system due to external shocks occur according to a phase-type renewal process. If the system is operational, the unit undergoes the effect of this shock. The time between two consecutive events is $P H$ distributed with representation $(\gamma, \mathbf{L})$. The order of the matrix $\mathbf{L}$ is equal to $t$.

Assumption 4. If the unit is operational, an external shock produces one of three different effects: extreme failure (non-repairable), external cumulative damage or aggravation of internal degradation. 
Assumption 5. An extreme (non-repairable) failure occurs with a probability equal to $\omega^{0}$ after an external shock.

Assumption 6. External damage may pass through an indeterminate number of external degradation states, $d$, which are partitioned into minor damage states (the first $d_{1}$ states) and major damage states (states $d_{1}+1, \ldots, d$ ). If the external degradation state is $i$, then the external shock changes to state $j$ with probability $q_{i j}$. These probabilities are contained in the matrix $\mathbf{Q}$. A cumulative external damage threshold is reached from the external damage states after an external shock, which is reflected in the probability column vector $\mathbf{Q}^{0}$. If this threshold is reached, the unit undergoes a non-repairable failure. Prior to such an external shock, the unit is in external degradation state 1 (no damage due to external shock). The initial distribution for external damage when a unit is at its initial online situation $\boldsymbol{\omega}=(1,0, \ldots, 0)_{1 \times d}$.

Assumption 7. An external shock modifies the internal degradation state while the unit is operational. If the internal degradation state is $i$, then the external shock changes it to state $j$ with probability $w_{i j}$. These probabilities are included in matrix $\mathbf{W}$. An internal repairable failure may occur for this reason from any performance state with a probability column vector $\mathbf{W}^{0}$.

Assumption 8. When a repairable failure occurs from operational state $i$, the unit system is sent to the repair facility. The repair time required depends on the state $i$ and it is $P H$ distributed with representation $\left(\boldsymbol{\beta}^{c, i}, \mathbf{S}_{c, i}\right)$ with order $z_{c, i}$ for $i=1, \ldots, n$.

Assumption 9. While the unit is operational, random inspections may be made. The time between two consecutive inspections is $P H$ distributed with representation $(\mathbf{\eta}, \mathbf{M})$ with order $\varepsilon$. Assumption 10. If an inspection observes major internal damage (state $i$ ) or major external cumulative damage (state $j$ ) then the unit goes to the repair facility for preventive maintenance. The preventive maintenance time depends on these states and it is $P H$ distributed with representation $\left(\beta^{p, i, j}, \mathbf{S}_{p, i, j}\right)$ with order $z_{p, i, j}$ for $i=n_{1}+1, \ldots, n$ and $j=d_{1}+$ $1, \ldots, d$. We assume $i=0$ or $j=0$ if minor internal or external damage is observed respectively.

Assumption 11. When the online unit undergoes a non-repairable failure, it is replaced by an identical unit.

The operation of the system is shown in Fig. 1.

\subsection{The model}

The system described above is governed by a vector Markov process. The state space $E$ is composed of the macro-states $E=\left\{E^{1}, E^{2}, E^{3}\right\}$, where $E^{k}$ contains the phases when the unit is operational $(k=1)$, the unit is in corrective repair $(k=2)$ and the unit is in preventive maintenance $(k=3)$. The macro-states $E^{2}$ and $E^{3}$ are composed of macro-states $E^{2, i}$ and $E^{3, i, j}$ respectively depending on the state at which the system failed or was inspected repectively. The phases of these macro-states are given by

$$
\begin{aligned}
& E^{1}=\{(i, j, u, m) ; 1 \leq i \leq n, 1 \leq j \leq t, 1 \leq u \leq d, 1 \leq m \leq \varepsilon\}, \\
& E^{2}=\left\{E^{2, i} ; 1 \leq i \leq n\right\}, \\
& E^{2, i}=\left\{(j, a) ; 1 \leq j \leq t, 1 \leq a \leq z_{c, i}\right\}, \text { for } i=1, \ldots, n, \\
& E^{3}=\left\{E^{3, i, j} ;\left\{n_{1}+1 \leq i \leq n, j=0\right\},\left\{d_{1}+1 \leq j \leq d, i=0\right\},\left\{n_{1}+1 \leq i \leq n, d_{1}+1 \leq j \leq d\right\}\right\} \\
& E^{3, i, j}=\left\{(j, a) ; 1 \leq j \leq t, 1 \leq a \leq z_{p, i, j}\right\} .
\end{aligned}
$$




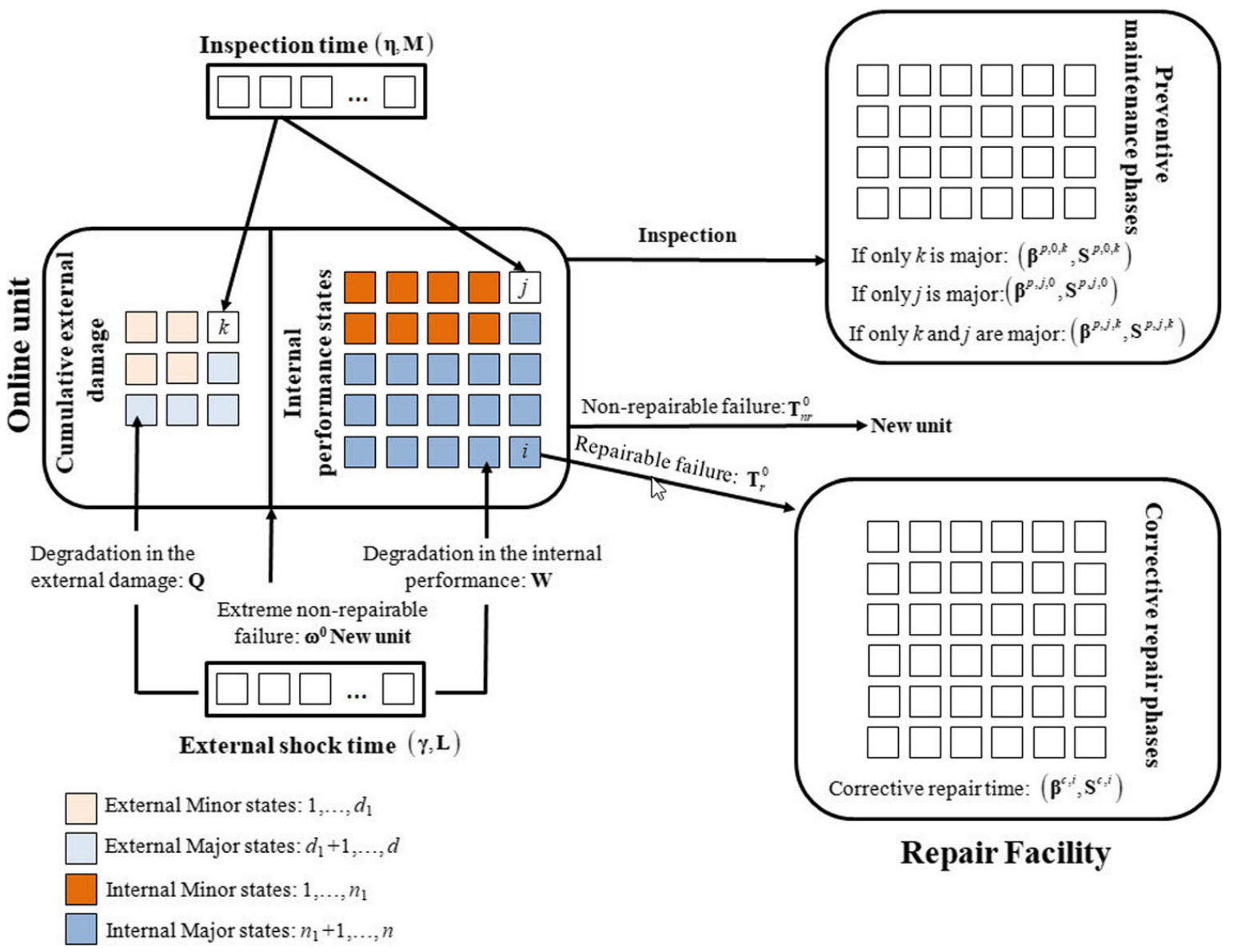

Fig. 1 Diagram of the system

where $i$ denotes the phase of the internal operational time, $j$ the phase of the external shock time, $u$ the cumulative external damage, $m$ the phase of the inspection time and $a$ the phase of the corrective repair or the preventive maintenance time.

When a complex system is subject to several types of events, it is important to analyse their behaviour in order to avoid or delay economic or catastrophic failures. The unit is subject to several types of events which may cause failures. Three different impacts on the online unit are considered; repairable internal failure $(A)$, inspection revealing major internal and/or external damage $(B)$ and non-repairable failure $(C)$.

The transition probabilities associated with these events are modelled in a well-structured way. Before discussing these probabilities, some auxiliary matrices are introduced.

The matrices $\mathbf{U}_{1}, \mathbf{U}_{2}^{i}$ and $\mathbf{V}_{1}, \mathbf{V}_{2}^{i}$ are square matrices of order $n$ and $d$ respectively, whose elements $(s, t)$ are given by,

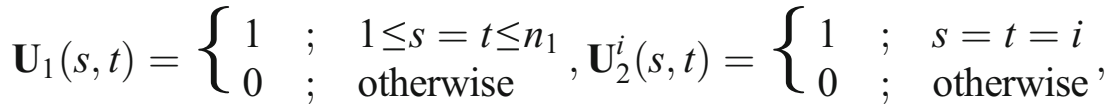

$$
\begin{aligned}
& \mathbf{V}_{1}(s, t)=\left\{\begin{array}{lll}
1 & ; & 1 \leq s=t \leq d_{1} \\
0 & ; & \text { otherwise }
\end{array}, \mathbf{V}_{2}^{i}(s, t)=\left\{\begin{array}{lll}
1 & ; & s=t=i \\
0 & ; & \text { otherwise }
\end{array}\right.\right.
\end{aligned}
$$

These matrices are applied when minor internal or cumulative external damage is observed by inspection ( $\mathbf{U}_{1}$ and $\mathbf{V}_{1}$, respectively), or when an event occurs specifically and exclusively during state $i\left(\mathbf{U}_{2}^{i}\right.$ and $\left.\mathbf{V}_{2}^{i}\right)$. 
Throughout the paper, given a matrix $\mathbf{A}$ we denote $\mathbf{A}^{0}$ to the matrix $\mathbf{A}^{0}=\mathbf{e}-\mathbf{A e}$, where $\mathbf{e}$ is a column vector of ones with appropriate order. The vector $\mathbf{e}_{a}$ denotes a vector of ones with order $a$. A matrix of zeros with appropriate order is denoted by $\mathbf{0}$. The symbol $\otimes$ denotes the Kronecker product.

The transition probabilities for the complex system, depending on the type of event (repairable failure, major damage revealed by inspection, non-repairable failure), are given as follows.

\subsubsection{No events}

These transitions take place when no events occur, with or without inspection (inspection only reveals minor damage). There are four possible outcomes:

a. No inspection is made (M), there is no external shock $(\mathbf{L})$ and the internal performance can modify its state without failure $(\mathbf{T})$.

b. No inspection is made (M) but an external shock takes place $\left(\mathbf{L}^{0} \boldsymbol{\gamma}\right)$ producing external damage without non-repairable failure $\left(\mathbf{Q}\left(1-\omega^{0}\right)\right)$ and the internal performance can be modified (TW).

c. An inspection is made $\left(\mathbf{M}^{0} \eta\right)$, no external shock takes place and the internal performance and external cumulative damage are in a minor damage state that can be modified without failure $\left(\mathbf{U}_{1} \mathbf{T} \otimes \mathbf{L} \otimes \mathbf{V}_{1}\right)$.

d. Inspection $\left(\mathbf{M}^{0} \eta\right)$ and external shock both take place. There is no failure. The external shock may provoke internal and/or external cumulative damage, but in either/both cases, inspection reveals the damage to be minor $\left(\mathbf{U}_{1} \mathbf{T W} \otimes \mathbf{L}^{0} \boldsymbol{\gamma} \otimes \mathbf{V}_{1} \mathbf{Q}\left(1-\omega^{0}\right)\right)$.

The transition probability matrix is

$$
\begin{aligned}
\mathbf{H}_{0} & =\left[\mathbf{T} \otimes \mathbf{L} \otimes \mathbf{I}+\mathbf{T W} \otimes \mathbf{L}^{0} \boldsymbol{\gamma} \otimes \mathbf{Q}\left(1-\omega^{0}\right)\right] \otimes \mathbf{M} \\
& +\left[\mathbf{U}_{1} \mathbf{T} \otimes \mathbf{L} \otimes \mathbf{V}_{1}+\mathbf{U}_{1} \mathbf{T} \mathbf{W} \otimes \mathbf{L}^{0} \boldsymbol{\gamma} \otimes \mathbf{V}_{1} \mathbf{Q}\left(1-\omega^{0}\right)\right] \otimes \mathbf{M}^{0} \eta
\end{aligned}
$$

\subsubsection{Internal repairable failure (a)}

The unit may undergo a repairable failure from the operational internal state $i$ due to wear or external shock. In the first case, this occurs because the repairable internal failure is produced from state $i\left(\mathbf{U}_{2}^{i} \mathbf{T}_{r}^{0}\right)$. In the second case, an internal failure may take place because a shock modifies the internal behaviour $\left(\mathbf{U}_{2}^{i} \mathbf{T} \mathbf{W}^{0}\right)$ without producing an extreme failure or causing the external threshold damage state to be reached $\left(\mathbf{Q e} \omega\left(1-\omega^{0}\right)\right)$.

The matrix that governs this transition is given by

$$
\mathbf{H}_{1}^{i}=\left[\mathbf{U}_{2}^{i} \mathbf{T}_{r}^{0} \otimes \mathbf{L} \otimes \mathbf{e}_{d}+\left(\mathbf{U}_{2}^{i} \mathbf{T}_{r}^{0}+\mathbf{U}_{2}^{i} \mathbf{T} \mathbf{W}^{0}\right) \otimes \mathbf{L}^{0} \boldsymbol{\gamma} \otimes \mathbf{Q e}\left(1-\omega^{0}\right)\right] \otimes \mathbf{e}_{\varepsilon}, \text { for } i=1, \ldots, n
$$

\subsubsection{Inspection reveals major internal and/or external damage (B)}

While the online unit is working, an inspection may take place. If it reveals any of the following situations, the unit must go the repair facility: 
a. Major internal damage from state $i$ without external shock, $\mathbf{U}_{2}^{i}\left(\mathbf{e}^{-} \mathbf{T}^{0}\right)$ and $\mathbf{V}_{1} \mathbf{e}$, or with external shock, $\mathbf{U}_{2}^{i} \mathbf{T W e}$ and $\mathbf{V}_{1} \mathbf{Q e}\left(1-\omega^{0}\right)$.

b. Major cumulative external damage from state $j$ without external shock, $\mathbf{U}_{1}\left(\mathbf{e}-\mathbf{T}^{0}\right)$ or $\mathbf{V}_{2}^{j} \mathbf{e}$, and with external shock, $\mathbf{U}_{1} \mathbf{T W e}$ and $\mathbf{V}_{2}^{j} \mathbf{Q e}\left(1-\omega^{0}\right)$.

c. Major internal and external cumulative damage from state $i$ and $j$, respectively, without external shock $\mathbf{U}_{2}^{i}\left(\mathbf{e}^{-} \mathbf{T}^{0}\right)$ and $\mathbf{V}_{2}^{j} \mathbf{e}$, or with external shock $\mathbf{U}_{2}^{i} \mathbf{T W e}$ and $\mathbf{V}_{2}^{j} \mathbf{Q e}\left(1-\omega^{0}\right)$.

Therefore,

$$
\begin{aligned}
& \mathbf{H}_{2}^{i, 0}=\left[\mathbf{U}_{2}^{i}\left(\mathbf{e}^{-} \mathbf{T}^{0}\right) \otimes \mathbf{L} \otimes \mathbf{V}_{1} \mathbf{e}+\mathbf{U}_{2}^{i} \mathbf{T W e} \otimes \mathbf{L}^{0} \boldsymbol{\gamma} \otimes \mathbf{V}_{1} \mathbf{Q e}\left(1-\omega^{0}\right)\right] \otimes \mathbf{M}^{0}, \\
& \mathbf{H}_{2}^{0, j}=\left[\mathbf{U}_{1}\left(\mathbf{e}^{-} \mathbf{T}^{0}\right) \otimes \mathbf{L} \otimes \mathbf{V}_{2}^{j} \mathbf{e}+\mathbf{U}_{1} \mathbf{T W e} \otimes \mathbf{L}^{0} \boldsymbol{\gamma} \otimes \mathbf{V}_{2}^{j} \mathbf{Q e}\left(1-\omega^{0}\right)\right] \otimes \mathbf{M}^{0}, \\
& \mathbf{H}_{2}^{i, j}=\left[\mathbf{U}_{2}^{i}\left(\mathbf{e}^{-} \mathbf{T}^{0}\right) \otimes \mathbf{L} \otimes \mathbf{V}_{2}^{j} \mathbf{e}+\mathbf{U}_{2}^{i} \mathbf{T W e} \otimes \mathbf{L}^{0} \boldsymbol{\gamma} \otimes \mathbf{V}_{2}^{j} \mathbf{Q e}\left(1-\omega^{0}\right)\right] \otimes \mathbf{M}^{0},
\end{aligned}
$$

for $i=n_{1}+1, \ldots, n$ and $j=d_{1}+1, \ldots, d$.

\subsubsection{Non-repairable failure (C)}

While the unit is working, a non-repairable failure may occur, due to wear from any internal operational state $\left(\mathbf{T}_{n r}^{0} \boldsymbol{\alpha}\right)$ or as a consequence of an external shock. This situation is arises when an external shock causes an extreme failure $\left(\omega^{0}\right)$ or when the cumulative external threshold is reached $\left(\mathbf{Q}^{0}\right)$. In either case, the operational time of the online unit, the cumulative external damage and the inspection time are all reinitialised $(\alpha, \omega, \eta)$. The matrix is given by

$$
\mathbf{H}_{3}=\left[\mathbf{T}_{n r}^{0} \boldsymbol{\alpha} \otimes\left[\mathbf{L} \otimes \mathbf{e} \boldsymbol{\omega}+\mathbf{L}^{0} \boldsymbol{\gamma} \otimes \mathbf{Q e} \boldsymbol{\omega}\left(1-\omega^{0}\right)\right]+\mathbf{e} \boldsymbol{\alpha} \otimes \mathbf{L}^{0} \boldsymbol{\gamma} \otimes\left(\mathbf{e} \boldsymbol{\omega} \omega^{0}+\mathbf{Q}^{0} \boldsymbol{\omega}\left(1-\omega^{0}\right)\right)\right] \otimes \mathbf{e \eta} .
$$

The state space and the model when preventive maintenance is not considered (case for $n_{1}=n$ and $d_{1}=d$ ) are described in the Appendix.

\section{The Markovian arrival process with marked arrivals}

The system described in Section 2 for the model with preventive maintenance is governed by the Markovian arrival process with marked arrivals (MMAP) with representation $\left(\boldsymbol{D}_{0}, \boldsymbol{D}_{1}, \boldsymbol{D}_{2}, \boldsymbol{D}_{3}\right)$, where $\mathbf{D}_{1}$ denotes the matrix associated with the repairable failure event, $\mathbf{D}_{2}$ denotes a major positive inspection with preventive maintenance and $\mathbf{D}_{3}$ denotes a non-repairable failure. The transition probability matrix of the Markov chain is given by $\boldsymbol{D}=\boldsymbol{D}_{0}+\boldsymbol{D}_{1}+\boldsymbol{D}_{2}+\boldsymbol{D}_{3}$. This matrix is built by considering the macrostates $E^{1}, E^{2}$ and $E^{3}$ given in Section 2.2.

\subsection{Matrix $\mathrm{D}_{0}$}

The matrix $\mathbf{D}_{0}$ contains the transitions when no failure or preventive maintenance take place. This matrix is given by 
$E^{1}$

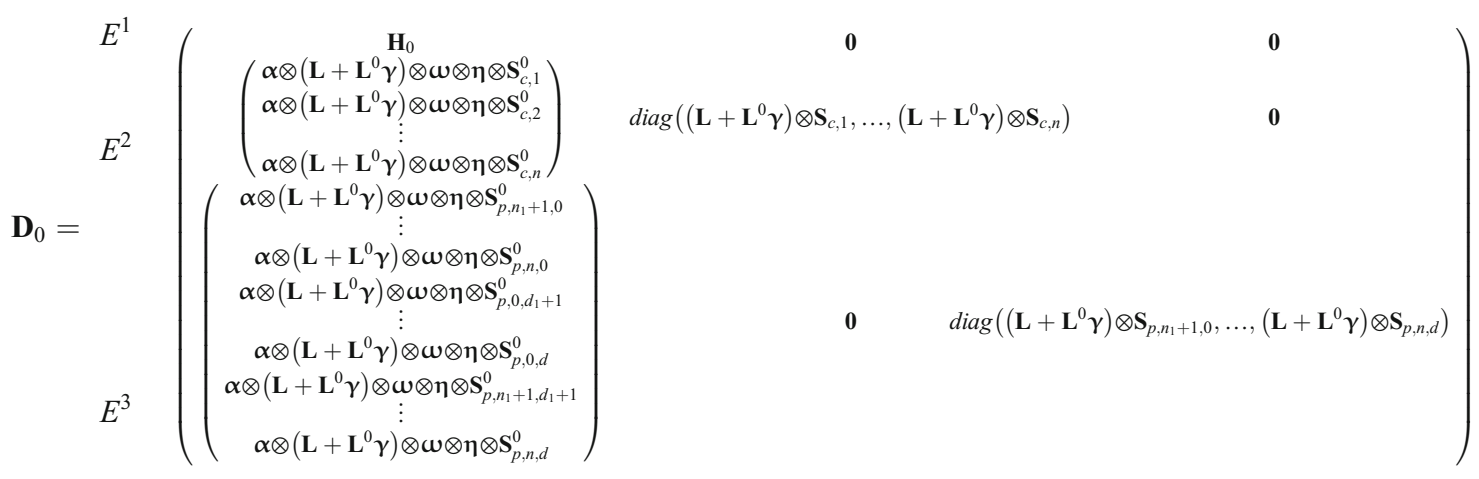

\subsection{Matrix $\mathrm{D}_{1}$}

The matrix $\mathbf{D}_{1}$ contains the transitions when a repairable internal failure occurs. This matrix is given by

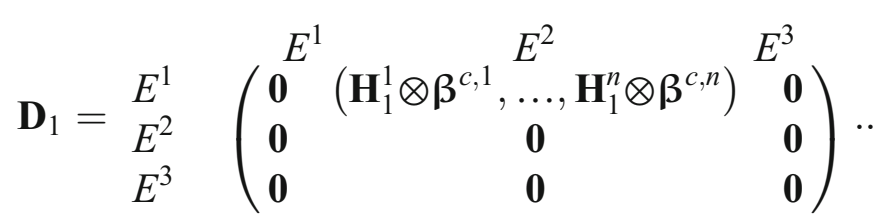

\subsection{Matrix $\mathrm{D}_{2}$}

The matrix $\mathbf{D}_{2}$ contains the transitions when preventive maintenance takes place. This matrix is given by

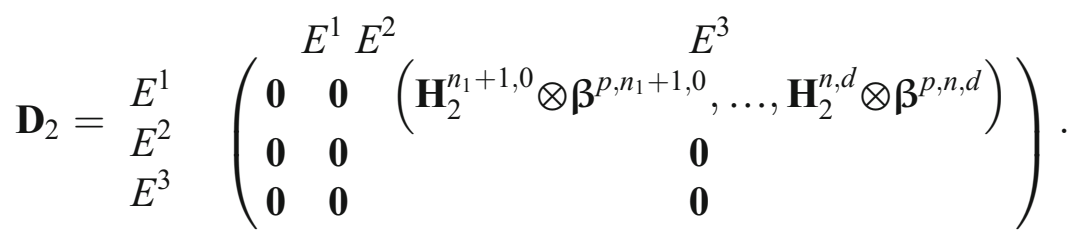

\subsection{Matrix $\mathrm{D}_{3}$}

The matrix $\mathbf{D}_{3}$ contains the transitions when a non-repairable failure occurs. This matrix is given by

$$
\left.\mathbf{D}_{3}=\begin{array}{cccc}
E^{1} & E^{2} & E^{3} \\
E^{2} & H^{3} & \mathbf{0} & \mathbf{0} \\
\mathbf{0} & \mathbf{0} & \mathbf{0} \\
\mathbf{0} & \mathbf{0} & \mathbf{0}
\end{array}\right)
$$




\section{Transient and stationary distributions}

The transition probabilities are presented in a computational, algorithmic way by considering matrix blocks. The transition probability matrix is given by

$$
\mathbf{D}=\mathbf{D}_{0}+\mathbf{D}_{1}+\mathbf{D}_{2}+\mathbf{D}_{3},
$$

and can be expressed by considering the blocks of the macro-states $E^{k}$, for $k=1,2,3$ as

$$
\left.\mathbf{D}=\begin{array}{llll}
E^{1} & E^{1} & E^{2} & E^{3} \\
E^{2} & \mathbf{D}_{11} & \mathbf{D}_{12} & \mathbf{D}_{13} \\
E^{3} & \mathbf{D}_{21} & \mathbf{D}_{22} & \mathbf{D}_{23}=\mathbf{0} \\
\mathbf{D}_{31} & \mathbf{D}_{32}=\mathbf{0} & \mathbf{D}_{33}
\end{array}\right) .
$$

External shocks may occur independently of whether the system is operational or not. For this reason, the initial distribution for the time of the external shock is the stationary distribution of the process with transition probability matrix $\boldsymbol{L}+\boldsymbol{L}^{0} \boldsymbol{\gamma}$. This stationary distribution is equal to

$$
\gamma^{*}=[1, \mathbf{0}]\left(e_{t} \mid\left(\mathbf{I}-\mathbf{L}-\mathbf{L}^{0} \gamma\right)^{*}\right)^{-1}
$$

where $\left(\mathbf{I}-\mathbf{L}-\mathbf{L}^{0} \boldsymbol{\gamma}\right)^{*}$ is the matrix $\mathbf{I}-\mathbf{L}-\mathbf{L}^{0} \boldsymbol{\gamma}$ without the first column.

The initial distribution of the system is given by

$$
\phi=\left(\boldsymbol{\alpha} \otimes \boldsymbol{\gamma}^{*} \otimes \boldsymbol{\omega} \otimes \boldsymbol{\eta}, \mathbf{0}\right),
$$

and so the transient distribution is given by $\mathbf{a}(\nu)=\phi \mathbf{D}^{\nu}$.

The transition probability matrix in $\nu$ steps has been calculated by matrix blocks to minimise the computational cost in a recursive form. Then,

$$
\mathbf{D}^{n}=\left(\begin{array}{lll}
\mathbf{D}_{11}^{(n)} & \mathbf{D}_{12}^{(n)} & \mathbf{D}_{13}^{(n)} \\
\mathbf{D}_{21}^{(n)} & \mathbf{D}_{22}^{(n)} & \mathbf{D}_{23}^{(n)} \\
\mathbf{D}_{31}^{(n)} & \mathbf{D}_{32}^{(n)} & \mathbf{D}_{33}^{(n)}
\end{array}\right),
$$

where

$$
\begin{aligned}
& \mathbf{D}_{i j}^{(1)}=\mathbf{D}_{i j} \\
& \mathbf{D}_{i j}^{(n)}=\sum_{\substack{k=1\\
}}^{\sum_{(k, j) \neq\{(2,3),(3,2)\}}^{3}} \mathbf{D}_{i k}^{(n-1)} \mathbf{D}_{k j} .
\end{aligned}
$$

The stationary distribution vector of $\mathbf{D}$ is denoted by $\theta$ and verifies $\theta \mathbf{D}=\theta$ and $\theta \mathbf{e}=1$. This stationary distribution is partitioned according to the macro-states, $\theta=\left(\theta_{1}, \theta_{2}, \theta_{3}\right)$, and it is calculated by considering matrix-analytic methods. The balance equations are expressed by blocks as

$$
\begin{gathered}
\theta_{1} \mathbf{D}_{11}+\theta_{2} \mathbf{D}_{21}+\theta_{3} \mathbf{D}_{31}=\theta_{1} \\
\theta_{1} \mathbf{D}_{12}+\theta_{2} \mathbf{D}_{22}=\theta_{2} \\
\theta_{1} \mathbf{D}_{13}+\theta_{3} \mathbf{D}_{33}=\theta_{3} .
\end{gathered}
$$


From (2) and (3)

$$
\begin{gathered}
\theta_{2}=\boldsymbol{\theta}_{1} \mathbf{D}_{12}\left(\mathbf{I}-\mathbf{D}_{22}\right)^{-1} \\
\boldsymbol{\theta}_{3}=\boldsymbol{\theta}_{1} \mathbf{D}_{13}\left(\mathbf{I}-\mathbf{D}_{33}\right)^{-1} .
\end{gathered}
$$

From (1), (2), (3) and from the normalization equation, $\theta_{1} \mathbf{e}+\theta_{2} \mathbf{e}+\theta_{3} \mathbf{e}=1$, we have that

$$
\begin{gathered}
\boldsymbol{\theta}_{1}\left[\mathbf{D}_{11}+\mathbf{D}_{12}\left(\mathbf{I}-\mathbf{D}_{22}\right)^{-1} \mathbf{D}_{21}+\mathbf{D}_{13}\left(\mathbf{I}-\mathbf{D}_{33}\right)^{-1} \mathbf{D}_{31}-\mathbf{I}\right]=\mathbf{0} \\
\boldsymbol{\theta}_{1}\left[\mathbf{I}+\mathbf{D}_{12}\left(\mathbf{I}-\mathbf{D}_{22}\right)^{-1}+\mathbf{D}_{13}\left(\mathbf{I}-\mathbf{D}_{33}\right)^{-1}\right] \mathbf{e}=1 .
\end{gathered}
$$

If we denote as $\mathbf{R}_{1}=\mathbf{D}_{11}+\mathbf{D}_{12}\left(\mathbf{I}-\mathbf{D}_{22}\right)^{-1} \mathbf{D}_{21}+\mathbf{D}_{13}\left(\mathbf{I}-\mathbf{D}_{33}\right)^{-1} \mathbf{D}_{31}-\mathbf{I}$ and $\mathbf{R}_{2}=\mathbf{e}_{n t d \varepsilon}+\mathbf{D}_{12}$ $\left(\mathbf{I}-\mathbf{D}_{22}\right)^{-1} \mathbf{e}_{t z 1}+\mathbf{D}_{13}\left(\mathbf{I}-\mathbf{D}_{33}\right)^{-1} \mathbf{e}_{t z_{2}}$ then

$$
\boldsymbol{\theta}_{1}=[1, \mathbf{0}]\left[\mathbf{R}_{2} \mid \mathbf{R}_{1}^{*}\right]^{-1},
$$

where $\mathbf{R}_{1}^{*}$ is the matrix $\mathbf{R}_{1}$ without the first column. Vectors $\theta_{2}$ and $\theta_{3}$ are obtained from (4) and (5) respectively.

\section{Measures: Availability, reliability and distribution of time between events}

The following measures associated with this system were determined.

\subsection{Availability}

The availability is the probability that the unit will be operational (macro-state $E^{1}$ ) at a certain time. If initially the system is operational then

$$
A(\nu)=\left(\boldsymbol{\alpha} \otimes \boldsymbol{\gamma}^{*} \otimes \boldsymbol{\omega} \otimes \boldsymbol{\eta}\right) \mathbf{D}_{11}^{(\nu)} \mathbf{e}
$$

The availability in the stationary regime is given by $A=\theta_{1} \mathbf{e}$.

\subsection{Reliability}

Regarding system reliability, various situations can be considered. The first is that of the time elapsed to first failure or preventive maintenance. This time is $P H$ distributed and is represented as $\left(\alpha \otimes \gamma^{*} \otimes \omega \otimes \eta, \mathbf{H}_{0}\right)$.

On the other hand, we may be interested in the time elapsed until the first time that the unit stops working. As non-repairable failures do not interrupt system performance, this time is $P H$ distributed, and is described as $\left(\boldsymbol{\alpha} \otimes \gamma^{*} \otimes \boldsymbol{\omega} \otimes \eta, \mathbf{D}_{11}\right)$. In both cases the reliability function is given by $R(\nu)=\left(\boldsymbol{\alpha} \otimes \boldsymbol{\gamma}^{*} \otimes \boldsymbol{\omega} \otimes \boldsymbol{\eta}\right) \mathbf{A}^{\nu} \mathbf{e}$, being $\mathbf{A}$ the matrix $\mathbf{H}_{0}$ or $\mathbf{D}_{11}$ respectively. 


\section{Mean number of events and correlations}

In this section, we consider the mean number of events associated with the Markovian arrival process with marked arrivals and correlations.

\subsection{Mean and variance of the number of events at a certain time}

Let $\left\{N_{h}(\nu) ; \nu \geq 0\right\}$ and $\{X(\nu) ; \nu \geq 0\}$ be the number of events of type $h \in C^{0}$ ( $C^{0}$ being the set composed of all types of events) and the underlying Markov process associated with the MMAP respectively.

To analyse the number of events in $[0, \nu]$, several functions must be defined. The probability distribution of the MMAP is given by the matrix

$$
\mathbf{P}\left(\left\{n_{h}, h \in C^{0}\right\}, \nu\right)=\left(P\left\{X(\nu)=j, N_{h}(\nu)=n_{h}, h \in C^{0} \mid X(0)=i\right\}\right) .
$$

The joint probability generating function for the number of arrivals in $[0, \nu]$ is defined as

$$
\mathbf{P}^{*}\left(\left\{z_{h}, h \in C^{0}\right\}, \nu\right)=\sum_{\left\{n_{h} \geq 0, h \in C^{0}\right\}} \mathbf{P}\left(\left\{n_{h}, h \in C^{0}\right\}, \nu\right) \prod_{h \in C^{0}} z_{h}^{n_{h}}
$$

and it is equal to

$$
\mathbf{P}^{*}(\mathbf{z}, \nu)=\left[\mathbf{D}^{*}(\mathbf{z})\right]^{\nu}
$$

where $\mathbf{D}^{*}(\mathbf{z})=\mathbf{D}^{*}\left(\left\{z_{h}, h \in C^{0}\right\}\right)=\mathbf{D}_{0}+\sum_{h \in C^{0}} z_{h} \mathbf{D}_{h}$.

It is well known that

$$
\left.\frac{\partial \mathbf{P}^{*}(\mathbf{z}, \nu)}{\partial z_{h}}\right|_{\mathbf{z}=(1, \ldots, 1)} \cdot \mathbf{e}=E\left[N_{h}(\nu)\right] \text { and }\left.\frac{\partial^{2} \mathbf{P}^{*}(\mathbf{z}, \nu)}{\partial z_{h}^{2}}\right|_{\mathbf{z}=(1, \ldots, 1)} \cdot \mathbf{e}=E\left[N_{h}(\nu)\left[N_{h}(\nu)-1\right]\right] \text { for } h \in C^{0}
$$

\subsubsection{Mean number of events}

The mean number of events type $h \in C^{0}$ up to time $\nu \geq 1$ is given by

$$
\begin{aligned}
E\left[N_{h}(\nu)\right]=\left.\frac{\partial \mathbf{P}^{*}(\mathbf{z}, \nu)}{\partial z_{h}}\right|_{\mathbf{z}=(1, \ldots, 1)} \mathbf{e} & =\left.\sum_{i=0}^{\nu-1}\left[\mathbf{D}^{*}(\mathbf{z})\right]^{i} \frac{\partial \mathbf{D}^{*}(\mathbf{z}, \nu)}{\partial z_{h}}\left[\mathbf{D}^{*}(\mathbf{z})\right]^{\nu-i-1}\right|_{\mathbf{z}=(1, \ldots, 1)} \mathbf{e} \\
& =\sum_{i=0}^{\nu-1} \mathbf{D}^{i} \mathbf{D}_{h} \mathbf{D}^{\nu-i-1} \mathbf{e}=\sum_{i=0}^{\nu-1} \mathbf{D}^{i} \mathbf{D}_{h} \mathbf{e},
\end{aligned}
$$

given that $\mathbf{D}$ is a stochastic matrix. If the initial distribution is given by $\phi$ then

$$
E_{\phi}\left[N_{h}(\nu)\right]=\phi \sum_{i=0}^{\nu-1} \mathbf{D}^{i} \mathbf{D}_{h} \mathbf{e}
$$

If the model is in the stationary regime then 


$$
E_{\theta}\left[N_{h}(\nu)\right]=\theta \sum_{i=0}^{\nu-1} \mathbf{D}^{i} \mathbf{D}_{h} \mathbf{e}=\nu \theta \mathbf{D}_{h} \mathbf{e}
$$

as $\theta$ verifies $\theta \mathbf{D}=\theta$.

\subsubsection{Variance}

The variance is obtained from the second partial derivative of the joint probability generating function with respect to $z_{h}$.

Thus, for $\nu \geq 2$

$$
\begin{aligned}
\frac{\partial^{2} \mathbf{P}^{*}(\mathbf{z}, \nu)}{\partial z_{h}^{2}} & =\frac{\partial^{2} \mathbf{P}^{*}(\mathbf{z}, \nu)}{\partial z_{h}^{2}}\left[\mathbf{D}^{*}(\mathbf{z})\right]^{\nu-1}+\frac{\partial \mathbf{D}^{*}(\mathbf{z})}{\partial z_{h}} \sum_{i=0}^{\nu-2}\left[\mathbf{D}^{*}(\mathbf{z})\right]^{i} \frac{\partial \mathbf{D}^{*}(\mathbf{z})}{\partial z_{h}}\left[\mathbf{D}^{*}(\mathbf{z})\right]^{\nu-i-2} \\
& +\sum_{i=1}^{\nu-2}\left[\sum_{j=0}^{i-1}\left[\mathbf{D}^{*}(\mathbf{z})\right]^{j} \frac{\partial \mathbf{D}^{*}(\mathbf{z})}{\partial z_{h}}\left[\mathbf{D}^{*}(\mathbf{z})\right]^{i-j-1} \frac{\partial \mathbf{D}^{*}(\mathbf{z})}{\partial z_{h}}\left[\mathbf{D}^{*}(\mathbf{z})\right]^{\nu-i-1}\right. \\
& +\left[\mathbf{D}^{*}(\mathbf{z})\right]^{i} \frac{\partial^{2} \mathbf{P}^{*}(\mathbf{z}, \nu)}{\partial z_{h}^{2}}\left[\mathbf{D}^{*}(\mathbf{z})\right]^{\nu-i-1} \\
& \left.+\left[\mathbf{D}^{*}(\mathbf{z})\right]^{i} \frac{\partial \mathbf{D}^{*}(\mathbf{z})}{\partial z_{h}} \sum_{j=0}^{\nu-i-2}\left[\mathbf{D}^{*}(\mathbf{z})\right]^{j} \frac{\partial \mathbf{D}^{*}(\mathbf{z})}{\partial z_{h}}\left[\mathbf{D}^{*}(\mathbf{z})\right]^{\nu-i-j-2}\right] \\
& +\sum_{j=0}^{\nu-2}\left[\mathbf{D}^{*}(\mathbf{z})\right]^{j} \frac{\partial \mathbf{D}^{*}(\mathbf{z})}{\partial z_{h}}\left[\mathbf{D}^{*}(\mathbf{z})\right]^{\nu-j-2} \frac{\partial \mathbf{D}^{*}(\mathbf{z})}{\partial z_{h}}+\left[\mathbf{D}^{*}(\mathbf{z})\right]^{\nu-1} \frac{\partial^{2} \mathbf{P}^{*}(\mathbf{z}, \nu)}{\partial z_{h}^{2}} .
\end{aligned}
$$

This function evaluated in $\mathbf{z}=(1, \ldots, 1)$ is equal to

$$
\begin{aligned}
\left.\frac{\partial^{2} \mathbf{P}^{*}(\mathbf{z}, \nu)}{\partial^{2} z_{h}}\right|_{\mathbf{z}=(1, \ldots, 1)} \mathbf{e} & =E\left[N_{h}(\nu)\left[N_{h}(\nu)-1\right]\right]= \\
& =\left[\mathbf{D}_{h} \sum_{i=0}^{\nu-2} \mathbf{D}^{i}+\sum_{i=1}^{\nu-2}\left[\sum_{j=0}^{i-1} \mathbf{D}^{j} \mathbf{D}_{h} \mathbf{D}^{i-j-1}+\mathbf{D}^{i} \mathbf{D}_{h} \sum_{j=0}^{\nu-i-2} \mathbf{D}^{j}\right]\right. \\
& \left.+\sum_{j=0}^{\nu-2} \mathbf{D}^{j} \mathbf{D}_{h} \mathbf{D}^{\nu-j-2}\right] \mathbf{D}_{h} \mathbf{e} .
\end{aligned}
$$

Given the initial distribution $\phi$, the variance is equal to

$$
\begin{gathered}
\operatorname{Var}_{\phi}\left[N_{h}(\nu)\right]=E_{\phi}\left[N_{h}(\nu)\left[N_{h}(\nu)-1\right]\right]+E_{\phi}\left[N_{h}(\nu)\right]-E_{\phi}\left[N_{h}(\nu)\right]^{2} \\
=\phi\left[\mathbf{D}_{h} \sum_{i=0}^{\nu-2} \mathbf{D}^{i}+\sum_{i=1}^{\nu-2}\left[\sum_{j=0}^{i-1} \mathbf{D}^{j} \mathbf{D}_{h} \mathbf{D}^{i-j-1}+\mathbf{D}^{i} \mathbf{D}_{h} \sum_{j=0}^{\nu-i-2} \mathbf{D}^{j}\right]+\sum_{j=0}^{\nu-2} \mathbf{D}^{j} \mathbf{D}_{h} \mathbf{D}^{\nu-j-2}\right] \mathbf{D}_{h} \mathbf{e} \\
+E_{\phi}\left[N_{h}(\nu)\right]-E_{\phi}\left[N_{h}(\nu)\right]^{2}
\end{gathered}
$$

If initially the system is in the stationary regime then,

$$
E_{\boldsymbol{\theta}}\left[N_{h}(\nu)\left[N_{h}(\nu)-1\right]\right]=2 \boldsymbol{\theta} \mathbf{D}_{h} \sum_{i=1}^{\nu-1} \sum_{j=0}^{i-1} \mathbf{D}^{j} \mathbf{D}_{h} \mathbf{e} .
$$

The variance for $N_{h}(\nu)$ is then given by 


$$
\begin{gathered}
\operatorname{Var}_{\boldsymbol{\theta}}\left[N_{h}(\nu)\right]=E_{\boldsymbol{\theta}}\left[N_{h}(\nu)\left[N_{h}(\nu)-1\right]\right]+E_{\boldsymbol{\theta}}\left[N_{h}(\nu)\right]-E_{\boldsymbol{\theta}}\left[N_{h}(\nu)\right]^{2} \\
=\boldsymbol{\theta}\left[\nu \mathbf{I}+2 \mathbf{D}_{h} \sum_{i=1}^{\nu-1} \sum_{j=0}^{i-1} \mathbf{D}^{j}\right] \mathbf{D}_{h} \mathbf{e}-\left(\nu \boldsymbol{\theta} \mathbf{D}_{h} \mathbf{e}\right)^{2} .
\end{gathered}
$$

\subsubsection{Covariance}

The covariance between $N_{h}(\nu)$ and $N_{k}(\nu)$ at time $\nu \geq 2$ for $k \neq h$ can be described as follows,

$$
\operatorname{Cov}_{\phi}\left(N_{h}(\nu), N_{k}(\nu)\right)=\frac{1}{2}\left\{\operatorname{Var}_{\phi}\left(N_{h}(\nu)+N_{k}(\nu)\right)-\operatorname{Var}_{\phi}\left(N_{h}(\nu)\right)-\operatorname{Var}_{\phi}\left(N_{k}(\nu)\right)\right\} .
$$

The process $N_{h}(\nu)+N_{k}(\nu)$ is the number of events type $h$ or $k$ in [0, $\left.\nu\right]$, therefore from (7)

$$
\begin{aligned}
\operatorname{Var}_{\phi}\left(N_{h}(\nu)+N_{k}(\nu)\right) & =\phi\left[\left(\mathbf{D}_{h}+\mathbf{D}_{k}\right) \sum_{i=0}^{\nu-2} \mathbf{D}^{i}+\sum_{i=1}^{\nu-2}\left[\sum_{j=0}^{i-1} \mathbf{D}^{j}\left(\mathbf{D}_{h}+\mathbf{D}_{k}\right) \mathbf{D}^{i-j-1}\right.\right. \\
& \left.\left.+\mathbf{D}^{i}\left(\mathbf{D}_{h}+\mathbf{D}_{k}\right) \sum_{j=0}^{\nu-i-2} \mathbf{D}^{j}\right]+\sum_{j=0}^{\nu-2} \mathbf{D}^{j}\left(\mathbf{D}_{h}+\mathbf{D}_{k}\right) \mathbf{D}^{\nu-j-2}\right]\left(\mathbf{D}_{h}+\mathbf{D}_{k}\right) \mathbf{e} \\
& +E_{\phi}\left[N_{h}(\nu)\right]+E_{\phi}\left[N_{k}(\nu)\right]-\left(E_{\phi}\left[N_{h}(\nu)\right]+E_{\phi}\left[N_{k}(\nu)\right]\right)^{2}
\end{aligned}
$$

For the stationary version of the MMAP we have from (8) that

$$
\operatorname{Var}_{\boldsymbol{\theta}}\left(N_{h}(\nu)+N_{k}(\nu)\right)=\boldsymbol{\theta}\left[\nu \mathbf{I}+2\left[\mathbf{D}_{h}+\mathbf{D}_{k}\right] \sum_{i=1}^{\nu-1} \sum_{j=0}^{i-1} \mathbf{D}^{j}\right]\left[\mathbf{D}_{h}+\mathbf{D}_{k}\right] \mathbf{e}-\left(\nu \boldsymbol{\theta}\left[\mathbf{D}_{h}+\mathbf{D}_{k}\right] \mathbf{e}\right)^{2}(.10)
$$

From (8), (9) and (10), this yields

$$
\begin{aligned}
\operatorname{Cov}_{\boldsymbol{\theta}}\left(N_{h}(\nu), N_{k}(\nu)\right)= & \boldsymbol{\theta}\left[\mathbf{D}_{h} \sum_{i=1}^{\nu-1} \sum_{j=0}^{i-1} \mathbf{D}^{j} \mathbf{D}_{k}+\mathbf{D}_{k} \sum_{i=1}^{\nu-1} \sum_{j=0}^{i-1} \mathbf{D}^{j} \mathbf{D}_{h}\right] \mathbf{e} \\
& +\frac{1}{2}\left[\left(\nu \theta \mathbf{D}_{k} \mathbf{e}\right)^{2}-\left(\nu \boldsymbol{\theta}\left[\mathbf{D}_{h}+\mathbf{D}_{k}\right] \mathbf{e}\right)^{2}+\left(\nu \boldsymbol{\theta} \mathbf{D}_{h} \mathbf{e}\right)^{2}\right] \\
& =\theta\left[\mathbf{D}_{h} \sum_{i=1}^{\nu-1} \sum_{j=0}^{i-1} \mathbf{D}^{j} \mathbf{D}_{k}+\mathbf{D}_{k} \sum_{i=1}^{\nu-1} \sum_{j=0}^{i-1} \mathbf{D}^{j} \mathbf{D}_{h}\right] \mathbf{e} \\
& -\nu^{2} \boldsymbol{\theta} \mathbf{D}_{h} \mathbf{e} \theta \mathbf{D}_{k} \mathbf{e}
\end{aligned}
$$

\subsubsection{Correlation coefficient functions}

The correlation coefficient at time $\nu$ between $N_{h}(\nu)$ and $N_{k}(\nu)$ is given by

$$
{ }_{\varphi} r_{h, k}(\nu)=\frac{\operatorname{Cov}_{\varphi}\left(N_{h}(\nu), N_{k}(\nu)\right)}{\sqrt{\operatorname{Var}_{\varphi}\left(N_{h}(\nu)\right) \operatorname{Var}_{\varphi}\left(N_{k}(\nu)\right)}},
$$

where $\varphi=\phi$ or $\varphi=\theta$ for the transient or stationary regime respectively.

The square of this value is the determination coefficient function. 


\subsection{Covariance between the numbers of events in non-overlapping intervals}

Let $\left\{N_{h}(\nu, \nu+\tau) ; \nu \geq 0\right\}$ be the number of events type $h$ in the interval $\left.] \nu, \nu+\tau\right]$, i.e. $N_{h}(\nu, \nu+$ $\tau)=N_{h}(\nu+\tau)-N_{h}(\nu)$. The numbers of events in non-overlapping intervals are correlated for a MMAP, but the numbers are conditionally independent, i.e. if $X(\nu)$ is known, then the number events process in $] \nu, \nu+\tau]$ is independent of that in $[0, \nu]$. Let the probability matrix be

$$
\begin{aligned}
& \mathbf{P}\left(\left\{n_{1 h}, h \in C^{0}\right\}, \nu,\left\{n_{2 k}, k \in C^{0}\right\}, \tau\right) \\
& =\left(P\left\{N_{h}(\nu)=n_{1 h}, N_{k}(\nu, \nu+\tau)=n_{2 k} ; h, k \in C^{0}, X(\nu+\tau)=j \mid X(0)=i\right\}\right),
\end{aligned}
$$

then, the joint probability generating function for the number of arrivals in $[0, \nu]$ and in $] \nu, \nu+$ $\tau]$ is defined as

$$
\begin{aligned}
& \mathbf{P}^{*}\left(\left\{z_{1 h}, h \in C^{0}\right\}, \nu,\left\{z_{2 k}, h \in C^{0}\right\}, \tau\right) \\
& =\sum_{\substack{\left\{n_{1 h} \geq 0, h \in C^{0}\right\} \\
\left\{n_{2 k} \geq 0, k \in C^{0}\right\}}} \mathbf{P}\left(\left\{n_{1 h}, h \in C^{0}\right\}, \nu,\left\{n_{2 k}, k \in C^{0}\right\}, \tau\right) \prod_{\substack{h \in C^{0} \\
k \in C^{0}}} z_{1 h}^{n_{1 h}} z_{2 h}^{n_{2 h}},
\end{aligned}
$$

and from the Markov property it is equal to

$$
\mathbf{P}^{*}\left(\left\{z_{1 h}, h \in C^{0}\right\}, \nu,\left\{z_{2 h}, h \in C^{0}\right\}, \tau\right)=\mathbf{P}^{*}\left(\mathbf{z}_{1}, \nu, \mathbf{z}_{2}, \tau\right)=\left[\mathbf{D}^{*}\left(\mathbf{z}_{1}\right)\right]^{\nu}\left[\mathbf{D}^{*}\left(\mathbf{z}_{2}\right)\right]^{\tau} .
$$

From this result,

$$
E_{\phi}\left[N_{h}(\nu) N_{k}(\nu, \nu+\tau)\right]=\left.\phi \frac{\partial^{2} \mathbf{P}^{*}\left(\mathbf{z}_{1}, \nu, \mathbf{z}_{2}, \tau\right)}{\partial z_{1 h} \partial z_{2 k}}\right|_{\mathbf{z}_{1}, \mathbf{z}_{2}=(1, \ldots, 1)} \mathbf{e}=\phi \sum_{i=0}^{\nu-1} \mathbf{D}^{i} \mathbf{D}_{h} \sum_{j=0}^{\tau-1} \mathbf{D}^{\nu+j-i-1} \mathbf{D}_{k} \mathbf{e},
$$

and then

$$
\operatorname{Cov}_{\phi}\left[N_{h}(\nu) N_{k}(\nu, \nu+\tau)\right]=\phi\left[\sum_{i=0}^{\nu-1} \mathbf{D}^{i} \mathbf{D}_{h}\left(\sum_{j=0}^{\tau-1} \mathbf{D}^{\nu+j-i-1}-\mathbf{e} \phi \sum_{j=\nu}^{\nu+\tau-1} \mathbf{D}^{j}\right)\right] \mathbf{D}_{k} \mathbf{e}
$$

If the system is initially in the stationary regime then

$$
\operatorname{Cov}_{\boldsymbol{\theta}}\left[N_{h}(\nu) N_{k}(\nu, \nu+\tau)\right]=\theta \sum_{i=0}^{\nu-1} \mathbf{D}_{h} \sum_{j=0}^{\tau-1} \mathbf{D}^{\nu+j-i-1} \mathbf{D}_{k} \mathbf{e}^{-\tau \nu} \boldsymbol{\theta} \mathbf{D}_{h} \mathbf{e} \boldsymbol{\Theta} \mathbf{D}_{k} \mathbf{e}
$$

\section{Rewards}

To optimise the performance of a system, it is useful to examine its work times, repair times, corrective and preventive maintenance actions, rewards and costs. The system described considers repairable and non-repairable failures and preventive maintenance. It is also interesting to examine whether preventive maintenance is economically profitable and what are the optimum states at which preventive maintenance should be applied after observation. 
In this analysis, we assume that the expected reward is equal to $b$ while the system is operational and that the expected costs while the unit is operational, per unit of time if the system is in a minor or major damage internal state, are equal to $c_{1}$ and $c_{2}$ respectively. While the system is in the repair facility, a cost depending on the state from where the unit has come is produced. We assume a cost equal to $c r_{i}^{a}$ per unit of time when it is in corrective repair phase $a$ from operational state $i$ and $p r_{i, j}^{a}$ per unit of time when it is in preventive maintenance phase $a$ from internal operational state $i$ and external cumulative damage $j$. These costs are arranged in the column vectors $\mathbf{c r}_{i}=\left(c r_{i}^{1}, \ldots, c r_{i}^{z_{c, i}}\right)^{\prime}$ and $\mathbf{p r}_{i, j}=\left(p r_{i, j}^{1}, \ldots, p r_{i, j}^{z_{p, i, j}}\right)^{\prime}$ respectively. A fixed expected cost, for one or more of various possible causes, is introduced for each time that a corrective repair or a preventive maintenance takes place, equal to $C R$ and $P M$ respectively.

While the unit is not operational the system experiences a loss equal to $A$ per unit of time. Finally, each new unit has a cost of $C$.

Figure 2 shows the complexity of costs and rewards.

\subsection{The net reward vector}

A net reward vector is built according to the state space described in Section 2.2. The net reward vector when the system is in the macro-state $E^{1}$ is given by

$$
\mathbf{n r}_{1}=b \mathbf{e}_{n t d \varepsilon}-\left(\begin{array}{c}
c_{1} \mathbf{e}_{n_{1}} \\
c_{2} \mathbf{e}_{n-n_{1}}
\end{array}\right) \otimes e_{t d \varepsilon}
$$

The cost vectors for the macro-states $E^{2}$ and $E^{3}$ (when the system is in corrective repair and in preventive maintenance respectively) are $\mathbf{n r}_{2}=-A \mathbf{e}_{t \sum_{i=1}^{n} z_{c, i}}-\left(\begin{array}{c}\mathbf{e}_{t} \otimes \mathbf{c r} \\ \vdots \\ \mathbf{e}_{t} \otimes \mathbf{c r}\end{array}\right)$ and

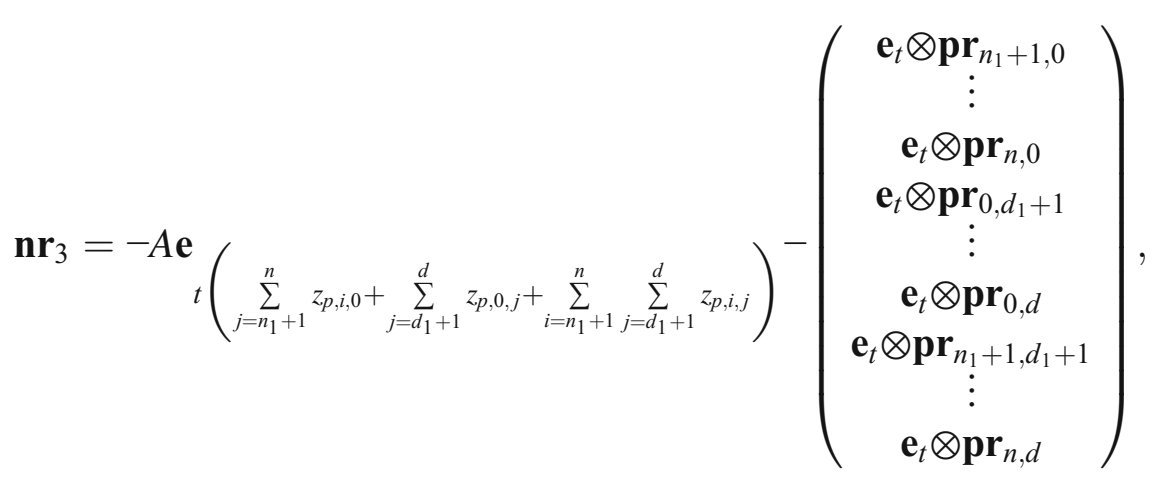

respectively.

Finally, the net reward vector associated to the state space is given by

$$
\mathbf{c}=\left(\begin{array}{l}
\mathbf{n} \mathbf{r}_{1} \\
\mathbf{n} \mathbf{r}_{2} \\
\mathbf{n} \mathbf{r}_{3}
\end{array}\right) \text {. }
$$




\section{COSTS AND REWARDS PER UNIT OF TIME}

- Expected reward while the system is operational: $b$

- Expected cost while the system is not operational: $A$

- Expected cost while the system is in an internal minor state: $c_{1}$

- Expected cost while the system is in an internal major state: $c_{2}$

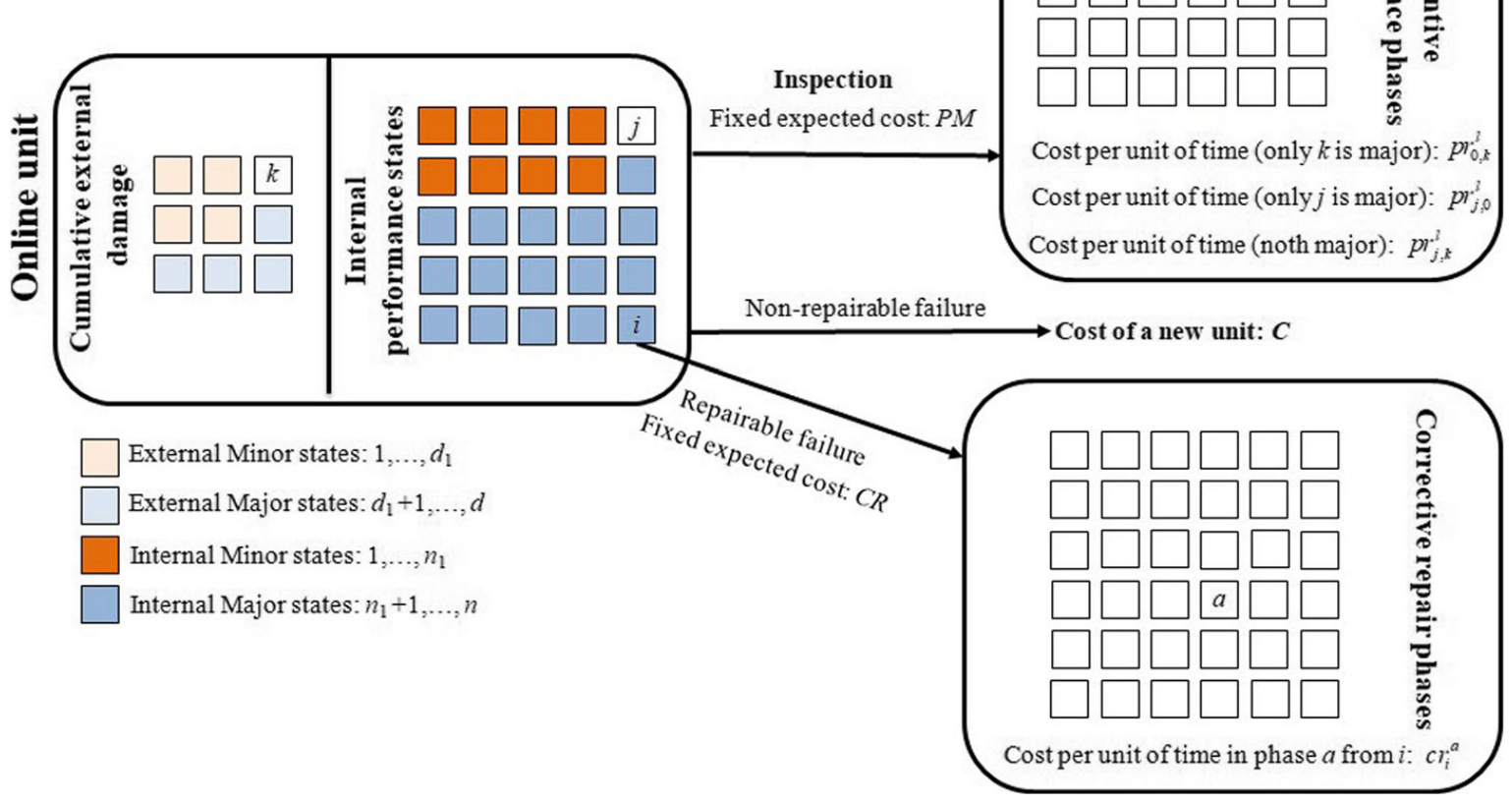

Fig. 2 Diagram of costs and rewards

\subsection{Expected net rewards}

An interesting aspect associated with the performance of a reliability system is that of the net reward per unit of time up to a certain time. This measure is composed of the expected net reward per unit of time minus the fixed cost for corrective repair, preventive maintenance and new units (the cost of the initial unit is included in this measure).

The cumulative expected net reward from the beginning up to time $\nu$ is given by

$$
\operatorname{ENR}_{\phi}(\nu)=\phi \sum_{n=0}^{\nu} \mathbf{D}^{n} \mathbf{c}-C R \cdot E_{\phi}\left[N_{1}(\nu)\right]-P M \cdot E_{\phi}\left[N_{2}(\nu)\right]-C \cdot\left(1+E_{\phi}\left[N_{3}(\nu)\right]\right) .
$$

Per unit of time this is equal to

$$
\Psi_{\phi}(\nu)=\frac{\operatorname{ENR}_{\phi}(\nu)}{\nu+1}
$$

If initially the system is in stationary regime then from (6) and (12),

$$
\operatorname{ENR}_{\boldsymbol{\theta}}(\nu)=(\nu+1) \theta \mathbf{c}-\nu \cdot C R \cdot \theta \mathbf{D}_{1} \mathbf{e}-\nu \cdot P M \cdot \theta \mathbf{D}_{2} \mathbf{e}-C \cdot\left(1+\nu \cdot \theta \mathbf{D}_{3} \mathbf{e}\right)
$$

and

$$
\Psi_{\theta}(\nu)=\theta \mathbf{c}-\frac{\nu \cdot C R}{\nu+1} \theta \mathbf{D}_{1} \mathbf{e}^{-} \frac{\nu \cdot P M}{\nu+1} \theta \mathbf{D}_{2} \mathbf{e}^{-} \frac{C}{\nu+1}\left(1+\nu \theta \mathbf{D}_{3} \mathbf{e}\right)
$$


Finally, independently of the initial distribution, the net reward per unit of time (steady state) is given by

$$
\Psi=\theta \mathbf{c}-C R \cdot \theta \mathbf{D}_{1} \mathbf{e}-P M \cdot \theta \mathbf{D}_{2} \mathbf{e}^{-} C \cdot\left(\theta \mathbf{D}_{3} \mathbf{e}\right) .
$$

\section{Preventive maintenance policy}

When preventive maintenance is introduced, the lifetime of the unit is of course extended, but at what price? Is this maintenance profitable? Multiple rewards and costs and corrective repair and preventive maintenance time distributions depend on the system state at which the unit failed and on the major damage state observed by inspection as it is shown in Figs. 1 and 2.

An important question is that of when preventive maintenance should be carried out, i.e. what is the threshold between minor and major damage? To answer this question, the expected net reward in the stationary regime should be taken into account. This measure is developed in (13). In fact, this function depends on the structure of the matrices from $n_{1}, d_{1}$. When an inspection takes place, the unit goes to the repair facility for preventive maintenance if the internal performance state and/or external cumulative damage observed are greater than $n_{1}$ and $d_{1}$ respectively.

Accordingly, a preventive maintenance policy is carried out adjusting $n_{1}$ and $d_{1}$ such that the net reward per unit of time, $\Psi\left(n_{1}, d_{1}\right)$ is maximum, for $n_{1}=1, \ldots, n$ and $d_{1}=1, \ldots, d$. Figure 3 shows a diagram of the maintenance policy.

Other measures could be taken into account such as reliability and availability, both of them depend on the barrier between minor and major internal and external damage, $n_{1}, d_{1}$, respectively.

\section{A numerical example}

This section highlights the value of preventive maintenance by comparing similar systems with and without preventive maintenance and with different maintenance policies, depending on the states at which inspection reveals major internal $\left(n_{1}\right)$ and external cumulative damage $\left(d_{1}\right)$. We assume a system composed of a generating set in a facility that requires a reliable electrical supply. This generating set is subject to degradation and may fail for the same reasons as any motor, provoking either a total or a repairable failure. The generating set passes through various degradation stages, and an internal repairable or non-repairable failure may occur from any of the different states. In addition, this device is subject to external failures which can modify its internal behaviour or even produce a non-repairable failure. Random inspections take place and the level of internal degradation and of cumulative external damage is observed. Lifetime distributions for the repair and preventive maintenance times depend, logically, on the level of degradation at which the system failed or was inspected. We assume a system with five internal states. The phase-type distributions embedded in the system - internal failure time, time between two consecutive external shocks, inspection time, corrective repair and preventive maintenance times depending on the previous state - are shown in Tables 1, 2 and 3 . 


\section{TIMES, COSTS AND REWARDS PER UNIT OF TIME $n_{1}$ and $d_{1}$ to maximize the net reward per unit of time (steady state)?}

- Internal operation time: $(\boldsymbol{\alpha}, \mathbf{T})$

- Expected reward while the system is operational: $b$

- Expected cost while the system is not operational: $A$

- Expected cost while the system is in an internal minor state: $c_{1}$

- Expected cost while the system is in an internal major state: $c_{2}$

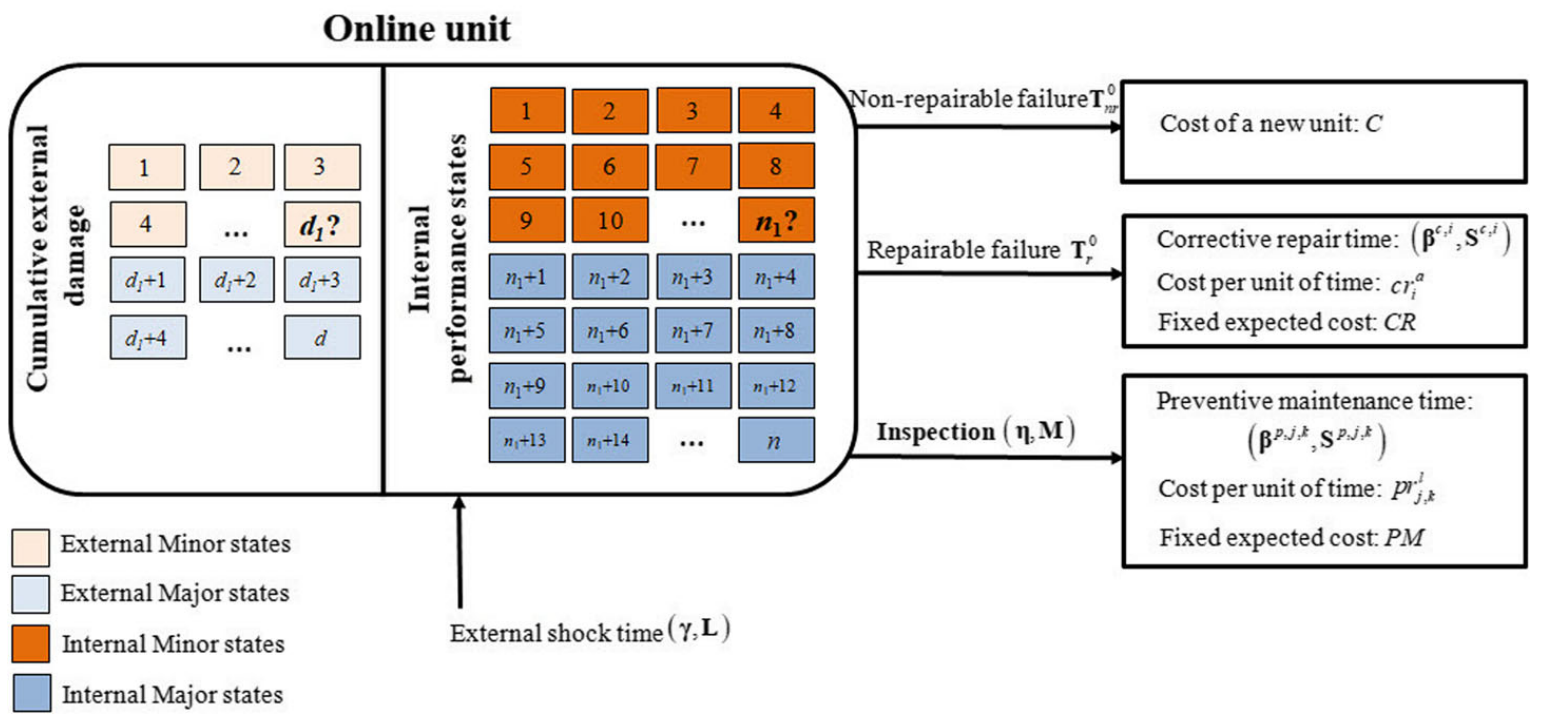

Fig. 3 Diagram of the preventive maintenance policy

Two types of internal failures are considered, repairable and non-repairable. The probability of either case occurring, from a transient state, is given by the column vectors $\boldsymbol{T}_{r}^{0}=$ $(0.008,0.008,0.195,0.195,0.1)^{\prime}$ and $\boldsymbol{T}_{n r}^{0}=(0.001,0.001,0.002,0.002,0.1)^{\prime}$ respectively. We assume that an external shock can produce an extreme non-repairable failure with probability 0.4 and that the matrix governing the transitions between cumulative episodes of external damage is

$$
\mathbf{Q}=\left(\begin{array}{cccc}
0 & 0.2 & 0.8 & 0 \\
0 & 0 & 0.5 & 0.5 \\
0 & 0 & 0 & 0.5 \\
0 & 0 & 0 & 0.3
\end{array}\right)
$$

Table 1 Internal failure, external shock and inspection phase-type distributions

\begin{tabular}{lccccc}
\hline Internal failure time & & External shock time & Inspection time \\
\hline $\boldsymbol{\alpha}=(1,0,0,0,0)$ & & $\gamma=(1,0)$ & $\boldsymbol{\eta}=(1,0,0)$ \\
$\mathbf{T}=\left(\begin{array}{ccccc}0.99 & 0.001 & 0 & 0 & 0 \\
0 & 0.99 & 0.001 & 0 & 0 \\
0 & 0 & 0.8 & 0.003 & 0 \\
0 & 0 & 0 & 0.8 & 0.003 \\
0 & 0 & 0 & 0 & 0.8\end{array}\right)$ & Mean time: 26.3780 & $\mathbf{M}=\left(\begin{array}{ccc}0.86 & 0.01 & 0.05 \\
0.8 & 0.04 & 0 \\
0.8 & 0.1 & 0.04\end{array}\right)$ \\
\\
\hline
\end{tabular}


When an external shock is produced, the cumulative threshold damage is reached from any transient state according to the column vector $\mathbf{Q}^{0}=(0,0,0.5,0.7)^{\prime}$. In this case a non-repairable failure occurs.

While the unit is operational and an external shock occurs, the internal state may be modified. The matrix governing this transition after an external shock is

$$
\mathbf{W}=\left(\begin{array}{ccccc}
0.6 & 0.2 & 0.1 & 0.1 & 0 \\
0 & 0.6 & 0.2 & 0.1 & 0.1 \\
0 & 0 & 0.6 & 0.2 & 0.2 \\
0 & 0 & 0 & 0.5 & 0.3 \\
0 & 0 & 0 & 0 & 0.4
\end{array}\right)
$$

From the matrix $\mathbf{W}$, it can be seen that if an external shock occurs then an internal repairable failure will take place only if the system is in state 4 or $5\left(\mathbf{W}^{0}=(0,0,0,0.2,0.6)^{\prime}\right)$.

\subsection{Rewards}

An interesting aspect regarding a complex reliability model subject to different types of failure and of repair (corrective and preventive), is to analyse the economic profit obtainable.

It is assumed that while the system is operational a reward equal to $b=10$ per unit of time is produced. However, while the system is active, a cost is also incurred. This cost per unit of time varies according to whether the system is working in a minor or a major state of internal damage. If the system is working in a minor damage state, a cost equal to 2 monetary units is incurred and this cost is 4 per unit of time if the system is in a major damage state.

If the system is in the repair facility, different costs arise. Each time the system undergoes a repairable failure, a fixed cost equal to $C R=10$ is incurred and a cost of $1,2,4,7$ or 10 monetary units per unit of time is incurred while the system is in corrective repair and if it failed from internal operational state 1,2, 3, 4 or 5 respectively. An analogous outcome is obtained for preventive maintenance. Each positive inspection provokes a fixed cost of 2 monetary units and the costs per unit of time in preventive maintenance are as shown in Table 4.

Finally, while the system is in the repair facility, economic losses of 10 monetary units per unit of time are incurred. Each new unit installed has a value equal to 200 monetary units.

The proportional time spent in each macro-state and the net reward per unit of time in the stationary regime from (13) for the different systems according to $d_{1}$ and $n_{1}$ are shown in Table 5.

Table 5 shows that the maximum net reward per unit of time is reached for $n_{1}=3$ and $d_{1}=$ 2. Then, given the operational time, the corrective repair times, the preventive maintenance times and the costs and rewards, the most profitable policy is to undertake preventive maintenance when the internal performance is in state 4 or 5 and when the cumulative external damage is in state 3 or 4 .

The proportional number of failures and major inspections per unit of time in the stationary regime were analysed for different systems according to $d_{1}$ and $n_{1}$. The values obtained are given in Table 6.

Next, we focus on the behaviour of the optimum model $n_{1}=3$ and $d_{1}=2$. If a new system, with an initial distribution of $\phi$ is considered, then a comparison can be drawn between the net 


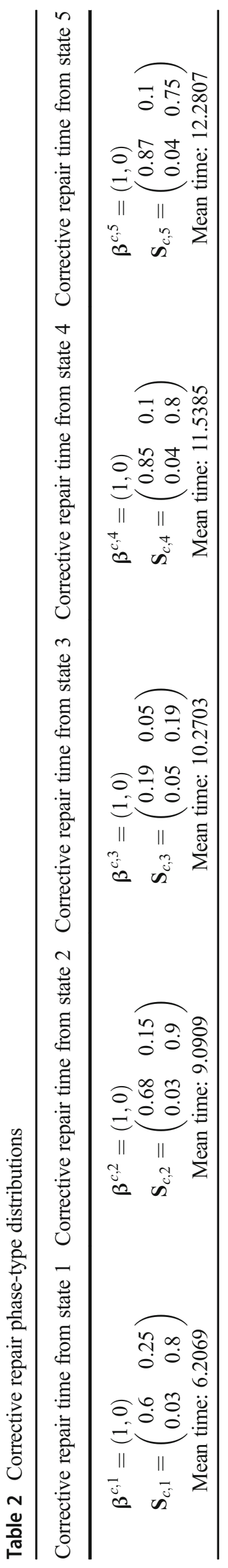


Table 3 Preventive maintenance phase-type distributions

Preventive maintenance time from Preventive maintenance time from Preventive maintenance time from only internal state $2 \quad$ only internal states 3 or 4

only internal state 5

$$
\begin{aligned}
& \boldsymbol{\beta}^{p, 2,0}=(1,0) \\
& \mathbf{S}_{p, 2,0}=\left(\begin{array}{ll}
0.02 & 0.02 \\
0.01 & 0.01
\end{array}\right)
\end{aligned}
$$

Mean time: 1.0412

Preventive maintenance time from only external cumulative damage

$\beta^{p, 0,2}=(1,0)$

$\mathbf{S}_{p, 0,2}=\left(\begin{array}{cc}0.01 & 0 \\ 0 & 0.01\end{array}\right)$

Mean time: 1.0101

Preventive maintenance time from internal state 2 and external cumulative damage 2

$$
\begin{aligned}
& \beta^{p, 2,2}=(1,0) \\
& \mathbf{S}_{p, 2,2}=\left(\begin{array}{cc}
0.4 & 0.15 \\
0.05 & 0.02
\end{array}\right)
\end{aligned}
$$

Mean time: 1.9466

Preventive maintenance time from internal state 3 or 4 and external cumulative damage 2

$$
\begin{aligned}
& \beta^{p, i, 2}=(1,0) \\
& \mathbf{S}_{p, i, 2}=\left(\begin{array}{ll}
0.3 & 0.2 \\
0.4 & 0.5
\end{array}\right) \\
& \text { Mean time: } 2.5926
\end{aligned}
$$

Preventive maintenance time from internal state 5 and external cumulative damage 2

$$
\begin{gathered}
\beta^{p, 5,2}=(1,0) \\
\mathbf{S}_{p, 5,2}=\left(\begin{array}{cc}
0.56 & 0.2 \\
0.2 & 0.4
\end{array}\right) \\
\text { Mean time: } 3.5714
\end{gathered}
$$

$$
\begin{aligned}
\beta^{p, i, 0} & =(1,0) \\
\mathbf{S}_{p, i, 0} & =\left(\begin{array}{ll}
0.3 & 0.2 \\
0.1 & 0.2
\end{array}\right)
\end{aligned}
$$

Mean time: 1.8519

Preventive maintenance time from only external cumulative damage 3

$\beta^{p, 0,3}=(1,0)$

$\mathbf{S}_{p, 0,3}=\left(\begin{array}{cc}0.02 & 0 \\ 0 & 0.02\end{array}\right)$

Mean time: 1.0204

Preventive maintenance time from internal state 2 and external cumulative damage 3

$\beta^{p, 2,3}=(1,0)$

$\mathbf{S}_{p, 2,3}=\left(\begin{array}{cc}0.4 & 0.2 \\ 0.01 & 0.01\end{array}\right)$

Mean time: 2.0101

Preventive maintenance time from internal state 3 or 4 and external cumulative damage 3

$\beta^{p, i, 3}=(1,0)$

$\mathbf{S}_{p, i, 3}=\left(\begin{array}{cc}0.56 & 0.1 \\ 0.3 & 0.1\end{array}\right)$

Mean time: 2.7322

Preventive maintenance time from internal state 5 and external cumulative damage 3

$$
\beta^{p, 5,3}=(1,0)
$$

$\mathbf{S}_{p, 5,3}=\left(\begin{array}{cc}0.58 & 0.2 \\ 0.15 & 0.45\end{array}\right)$

Mean time: 3.7313 $\beta^{p, 5,0}=(1,0)$

$\mathbf{S}_{p, 5,0}=\left(\begin{array}{cc}0.5 & 0.2 \\ 0.1 & 0.4\end{array}\right)$

Mean time: 2.8571

Preventive maintenance time from only external cumulative damage 4

$\beta^{p, 0,4}=(1,0)$

$\mathbf{S}_{p, 0,4}=\left(\begin{array}{cc}0.2 & 0.01 \\ 0.02 & 0.1\end{array}\right)$

Mean time: 1.2642

Preventive maintenance time from internal state 2 and external cumulative damage 4

$\beta^{p, 2,4}=(1,0)$

$\mathbf{S}_{p, 2,4}=\left(\begin{array}{cc}0.5 & 0.05 \\ 0.05 & 0.02\end{array}\right)$

Mean time: 2.1128

Preventive maintenance time from internal state 3 or 4 and external cumulative damage 4

$\beta^{p, i, 4}=(1,0)$

$\mathbf{S}_{p, i, 4}=\left(\begin{array}{cc}0.3 & 0.5 \\ 0.25 & 0.2\end{array}\right)$

Mean time: 2.9885

Preventive maintenance time from internal state 5 and external cumulative damage 4

$\beta^{p, 5,4}=(1,0)$

$\mathbf{S}_{p, 5,4}=\left(\begin{array}{cc}0.62 & 0.2 \\ 0.2 & 0.45\end{array}\right)$

Mean time: 4.4379

reward per unit of time for this optimum model and one without preventive maintenance. Figure 4 shows the net reward up to a certain time and per unit of time for both models (optimum and without preventive maintenance).

For both models, the expected net reward up to a certain time increases with time, and both models are loss-making up to a certain time. The optimum system with (without) preventive

Table 4 Cost per unit of time when the unit goes to the repair facility after inspection has revealed major internal damage state $i$ and major external cumulative damage $j$ ( 0 indicates minor damage on inspection)

$$
\text { States observed by inspection }(i, j)
$$

Monetary units per unit of time if the unit goes to repair facility

States observed by inspection $(i, j)$

Monetary units per unit of time if the unit goes to repair facility

$$
\begin{array}{lllllll}
(2,0) & (3,0) & (5,0) & (0,2) & (0,3) & (2,2) & (2,3) \\
& (4,0) & & & (0,4) & & (2,4)
\end{array}
$$

$\begin{array}{lllllll}0.2 & 0.5 & 1 & 0.1 & 1 & 0.2 & 1.1\end{array}$

$(3,2) \quad(3,3) \quad(3,4) \quad(4,2) \quad(4,3) \quad(5,2) \quad(5,3)$ $(4,4) \quad(5,4)$

$\begin{array}{lllllll}0.6 & 1.5 & 1.2 & 0.5 & 1 & 0.9 & 2\end{array}$


Table 5 Proportional time spent in each macro-state and net reward per unit of time in the stationary regime. The optimum value in bold

\begin{tabular}{|c|c|c|c|c|}
\hline & $\theta_{1} \cdot \mathbf{e}$ & $\theta_{2} \cdot \mathbf{e}$ & $\theta_{3} \cdot \mathbf{e}$ & $\Psi\left(n_{1}, d_{1}\right)$ \\
\hline$d_{1}=1 ; n_{1}=1$ & 0.9222 & 0.0625 & 0.0153 & 3.0492 \\
\hline$d_{1}=1 ; n_{1}=2$ & 0.9246 & 0.0630 & 0.0124 & 3.0830 \\
\hline$d_{1}=1 ; n_{1}=3$ & 0.9253 & 0.0630 & 0.0116 & 3.0940 \\
\hline$d_{1}=1 ; n_{1}=4$ & 0.9260 & 0.0631 & 0.0109 & 3.1041 \\
\hline$d_{1}=1 ; n_{1}=5$ & 0.9261 & 0.0631 & 0.0108 & 3.1054 \\
\hline$d_{1}=2 ; n_{1}=1$ & 0.9239 & 0.0626 & 0.0136 & 3.0709 \\
\hline$d_{1}=2 ; n_{1}=2$ & 0.9260 & 0.0634 & 0.0106 & 3.0965 \\
\hline$d_{1}=2 ; n_{1}=3$ & 0.9266 & 0.0636 & 0.0098 & 3.1056 \\
\hline$d_{1}=2 ; n_{1}=4$ & 0.9266 & 0.0643 & 0.0091 & 3.1004 \\
\hline$d_{1}=2 ; n_{1}=5$ & 0.9267 & 0.0643 & 0.0090 & 3.1014 \\
\hline$d_{1}=3 ; n_{1}=1$ & 0.9316 & 0.0621 & 0.0063 & 2.9890 \\
\hline$d_{1}=3 ; n_{1}=2$ & 0.9328 & 0.0635 & 0.0036 & 2.9329 \\
\hline$d_{1}=3 ; n_{1}=3$ & 0.9332 & 0.0640 & 0.0028 & 2.9322 \\
\hline$d_{1}=3 ; n_{1}=4$ & 0.9308 & 0.0670 & 0.0022 & 2.8701 \\
\hline$d_{1}=3 ; n_{1}=5$ & 0.9310 & 0.0670 & 0.0020 & 2.8726 \\
\hline$d_{1}=4 ; n_{1}=1$ & 0.9334 & 0.0620 & 0.0046 & 2.9690 \\
\hline$d_{1}=4 ; n_{1}=2$ & 0.9347 & 0.0636 & 0.0017 & 2.8836 \\
\hline$d_{1}=4 ; n_{1}=3$ & 0.9351 & 0.0641 & 0.0008 & 2.8797 \\
\hline$d_{1}=4 ; n_{1}=4$ & 0.9322 & 0.0676 & 0.0001 & 2.8078 \\
\hline$d_{1}=4 ; n_{1}=5$ & 0.9321 & 0.0679 & 0 & 2.8003 \\
\hline
\end{tabular}

maintenance incurs losses up to time 60 (63). The expected profit from the start until this time is $2.4332(2.2585)$ and, from then on, the expected profit per unit of time from the start is equal to $0.0406(0.0358)$. Taking into account the transient analysis, the expected net reward up to time 1000 for the optimum model with preventive maintenance is equal to 2921.7 , in contrast to the 2626.5 for the model without preventive maintenance.

Table 6 Proportional number of repairable and non-repairable failures and preventive maintenance per unit of time in stationary regime

\begin{tabular}{llll}
\hline & Repairable failure ratio & Major inspection ratio & Non-repairable failure ratio \\
\hline$d_{1}=1 ; n_{1}=1$ & 0.0098 & 0.0109 & 0.0163 \\
$d_{1}=1 ; n_{1}=2$ & 0.0099 & 0.0105 & 0.0163 \\
$d_{1}=1 ; n_{1}=3$ & 0.0099 & 0.0105 & 0.0163 \\
$d_{1}=1 ; n_{1}=4$ & 0.0099 & 0.0105 & 0.0163 \\
$d_{1}=1 ; n_{1}=5$ & 0.0099 & 0.0105 & 0.0163 \\
$d_{1}=2 ; n_{1}=1$ & 0.0098 & 0.0097 & 0.0163 \\
$d_{1}=2 ; n_{1}=2$ & 0.0099 & 0.0088 & 0.0164 \\
$d_{1}=2 ; n_{1}=3$ & 0.0100 & 0.0087 & 0.0164 \\
$d_{1}=2 ; n_{1}=4$ & 0.0100 & 0.0086 & 0.0164 \\
$d_{1}=2 ; n_{1}=5$ & 0.0101 & 0.0086 & 0.0164 \\
$d_{1}=3 ; n_{1}=1$ & 0.0098 & 0.0046 & 0.0175 \\
$d_{1}=3 ; n_{1}=2$ & 0.0098 & 0.0023 & 0.0179 \\
$d_{1}=3 ; n_{1}=3$ & 0.0101 & 0.0019 & 0.0179 \\
$d_{1}=3 ; n_{1}=4$ & 0.0104 & 0.0016 & 0.0179 \\
$d_{1}=3 ; n_{1}=5$ & 0.0104 & 0.0016 & 0.0179 \\
$d_{1}=4 ; n_{1}=1$ & 0.0097 & 0.0038 & 0.0178 \\
$d_{1}=4 ; n_{1}=2$ & 0.0098 & 0.0009 & 0.0184 \\
$d_{1}=4 ; n_{1}=3$ & 0.0102 & 0.0004 & 0.0184 \\
$d_{1}=4 ; n_{1}=4$ & 0.0105 & 0.0000 & 0.0184 \\
$d_{1}=4 ; n_{1}=5$ & 0.0105 & $* * * *$ & 0.0184
\end{tabular}



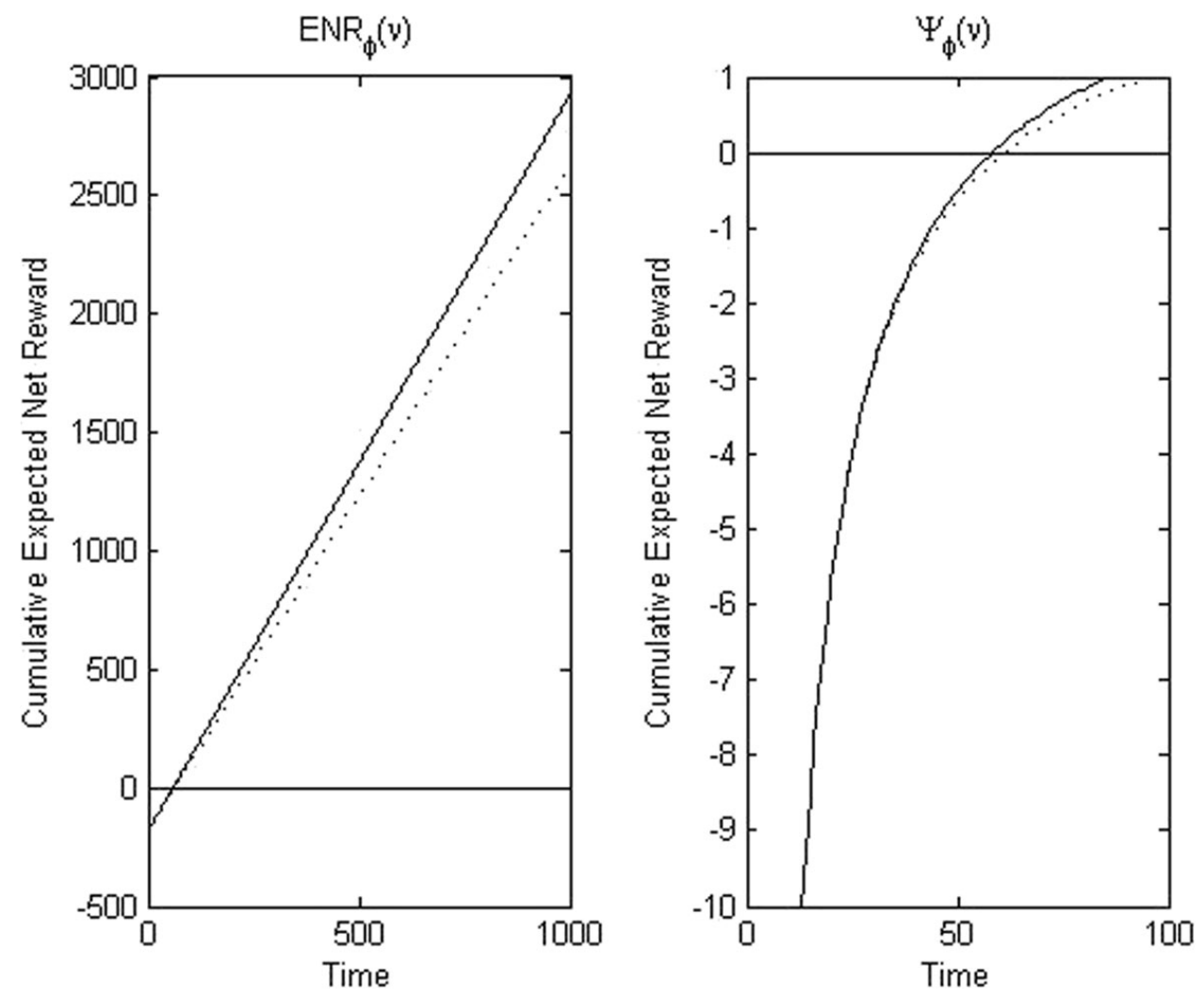

Fig. 4 Expected net reward up to a certain time and per unit of time (with preventive maintenance, continuous line; without preventive maintenance, dashed line)

After including the times, several measures were calculated. Table 7 shows the probability of the system being in any of the macro-states (operational, corrective repair or preventive maintenance) at various times for each model: optimum and without preventive maintenance.

The mean number of events is described in Section 6. If we assume that the model is in a stationary regime, then the mean number of repairable and non-repairable failures and the mean number of major inspection events for both models can be calculated from (6). These details are shown in Table 8.

The difference between the number of repairable and non-repairable failures is considerable. Thus, up to time 1000 the mean number of non-repairable failures decreases by almost two units and the mean number of repairable failures decreases by half a unit.

Table 7 Probability of the system being in each macro-state (in parentheses, the optimum model without preventive maintenance)

\begin{tabular}{cllr}
\hline Time $(\nu)$ & $E_{1}$ & $E_{2}$ & $E_{3}$ \\
\hline \multirow{2}{*}{10} & 0.9440 & 0.0485 & 0.0075 \\
& $(0.9500)$ & $(0.0500)$ & 0.0093 \\
20 & 0.9313 & 0.0594 & 0.0098 \\
& $(0.9371)$ & $(0.0629)$ & 0.0098 \\
50 & 0.9270 & $(0.0676)$ & 0.0632 \\
\multirow{2}{*}{100} & $(0.9324)$ & 0.035 & 0.0098 \\
& $(0.9266$ & $(0.0678)$ & 0.0636 \\
\end{tabular}


Table 8 Mean times of events up to a certain time (in parentheses the model without preventive maintenance)

\begin{tabular}{cllc}
\hline Time $(\nu)$ & Repairable failure $E_{\theta}\left[N_{1}(\nu)\right]$ & Preventive maintenance $E_{\theta}\left[N_{2}(\nu)\right]$ & Non-repairable failure $E_{\theta}\left[N_{3}(\nu)\right]$ \\
\hline \multirow{2}{*}{50} & 0.4954 & 0.3757 & 0.8180 \\
& $(0.5220)$ & & $(0.8716)$ \\
\multirow{2}{*}{100} & 0.9947 & 0.8095 & 1.6370 \\
& $(1.0470)$ & & $(1.7858)$ \\
200 & 1.9934 & 1.6771 & 3.2749 \\
& $(2.0957)$ & & $(3.6244)$ \\
500 & 4.9895 & 4.2799 & 8.1888 \\
& $(5.2419)$ & & $(9.1379)$ \\
1000 & 9.9830 & 8.6180 & 16.3786 \\
& $(10.4854)$ & & $(18.3270)$ \\
\hline
\end{tabular}

The correlation between the numbers of events is also described. Figure 5 shows the correlation coefficient function obtained from (11).

All correlations between events decrease with time and are negative. The events repairable failure, preventive maintenance and non-repairable failure are denoted by 1, 2 and 3 respectively. In the optimum model with preventive maintenance, the maximum negative correlation occurs between repairable failure and preventive maintenance. Comparison of the models with and without preventive maintenance shows that the negative correlation between repairable and non-repairable failures decreases in both cases, but is considerably larger for the second model. The correlation between the number of repairable failures and non-repairable failures at time 1000 is equal to -0.1083 for the model with preventive maintenance and -0.1649 for the model without preventive maintenance.
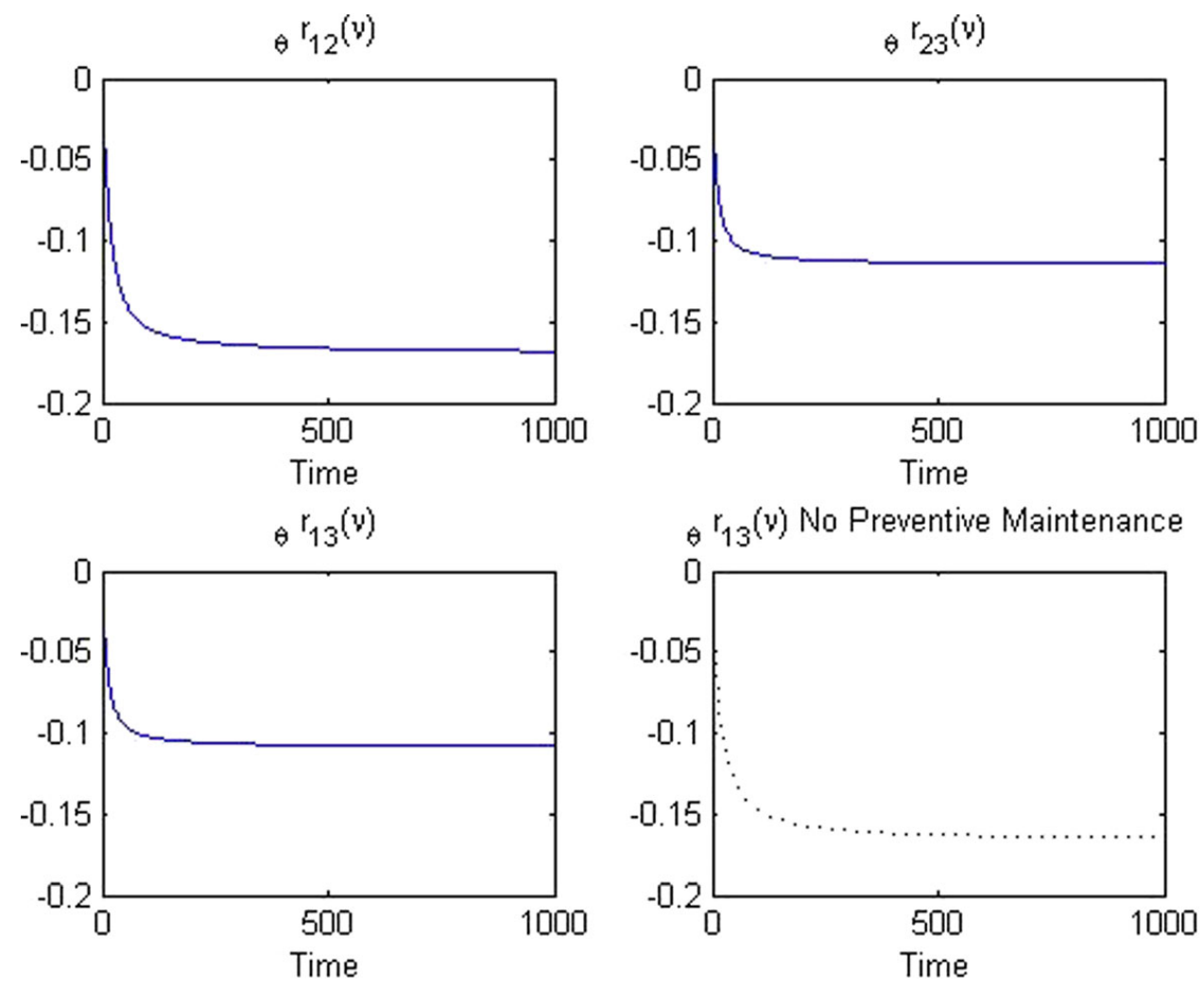

Fig. 5 Correlation Coefficient function (model with preventive maintenance, continuous line; model without preventive maintenance, dashed line) 


\section{Conclusions}

This paper presents a complex multi-state model subject to various types of failure, external shocks and preventive maintenance, modelled using a Markovian arrival process with marked arrivals, in an algorithmic and computational form. Deterioration of the system can produce repairable and/or nonrepairable failures. When a repairable failure takes place, the unit goes to the repair facility for corrective repair. If the failure is non-repairable, the unit is replaced by a new unit. Various events may occur following a renewal process and if the system is operational, cumulative damage may be produced, such as degradation of the internal performance or extreme failure (non-repairable). The internal performance of the system and cumulative external damage are partitioned into minor and major levels or states. Random inspections are conducted and preventive maintenance is performed if major internal and/or external cumulative damage is observed. Different time distributions are assumed for corrective repair and preventive maintenance depending on the state of the unit when the event occurs.

This complex system is modelled by a MMAP, which is shown to be useful for expressing the modelling and its associated measures in a well-structured form. Furthermore, this method makes it possible to determine the transient and stationary distributions and measures associated with the system in a matrix-algorithmic and computational form. The mean number of events and the variance up to a certain time are also calculated. The numbers of different events up to a certain time are correlated and are determined in an algorithmic form. Costs and rewards are included in the model to analyse the effectiveness of preventive maintenance from an economic standpoint, and depend on the state of the unit when it goes to the repair facility. This study considers whether preventive maintenance is profitable, and also shows how the system can be optimised according to its internal performance and the external cumulative damage states revealed by inspection. A numerical example, optimising the system by determining the optimum states from an economic standpoint, illustrates the versatility of the model proposed.

Acknowledgements This paper is partially supported by the Junta de Andalucía, Spain, under the grant FQM307 and by the Ministerio de Economía y Competitividad, España, under Grant MTM2017-88708-P and by the European Regional Development Fund (ERDF).

\section{Appendix}

In a similar way to the procedure described in Section 2.2, the state space and events are built for the model without preventive maintenance, $n_{1}=n$ and $d_{1}=d$. In this case, the state space $E$ is composed of the macro-states $E=\left\{E^{1}, E^{2}\right\}$, where $E^{k}$ contains the phases when the unit is operational $(k=1)$ and the unit is in corrective repair $(k=2)$. The phases are given by

$$
\begin{aligned}
& E^{1}=\{(i, j, u, m) ; 1 \leq i \leq n, 1 \leq j \leq t, 1 \leq u \leq d, 1 \leq m \leq \varepsilon\}, \\
& E^{2}=\left\{E^{2, i} ; 1 \leq i \leq n\right\}, \\
& E^{2, i}=\left\{(j, a) ; 1 \leq j \leq t, 1 \leq a \leq z_{c, i}\right\}, \text { for } i=1, \ldots, n,
\end{aligned}
$$

For this new situation the matrices are given by

$$
\begin{aligned}
& \mathbf{H}_{0}=\mathbf{T} \otimes \mathbf{L} \otimes \mathbf{I}+\mathbf{T W} \otimes \mathbf{L}^{0} \boldsymbol{\gamma} \otimes \mathbf{Q}\left(1-\omega^{0}\right) \\
& \mathbf{H}_{1}^{i}=\mathbf{U}_{2}^{i} \mathbf{T}_{r}^{0} \otimes \mathbf{L} \otimes \mathbf{e}_{d}+\left(\mathbf{U}_{2}^{i} \mathbf{T}_{r}^{0}+\mathbf{U}_{2}^{i} \mathbf{T} \mathbf{W}^{0}\right) \otimes \mathbf{L}^{0} \boldsymbol{\gamma} \otimes \mathbf{Q e}\left(1-\omega^{0}\right) ; i=1, \ldots, n . \\
& \mathbf{H}_{3}=\mathbf{T}_{n r}^{0} \boldsymbol{\alpha} \otimes\left[\mathbf{L} \otimes \mathbf{e} \boldsymbol{\omega}+\mathbf{L}^{0} \boldsymbol{\gamma} \otimes \mathbf{Q e \omega}\left(1-\omega^{0}\right)\right]+\mathbf{e} \boldsymbol{\alpha} \otimes \mathbf{L}^{0} \boldsymbol{\gamma} \otimes\left(\mathbf{e} \boldsymbol{\omega} \omega^{0}+\mathbf{Q}^{0} \boldsymbol{\omega}\left(1-\omega^{0}\right)\right) .
\end{aligned}
$$


Publisher's Note Springer Nature remains neutral with regard to jurisdictional claims in published maps and institutional affiliations.

\section{References}

Buchholz P, Kriege J, Felko I (2014) Input modeling with phase-type distributions and Markov models. Theory and applications. Springer, Heidelberg

Dewanji A, Segupta D, Chakraborty K (2011) A discrete time model for software reliability with application to a flight control software. Appl Stoch Model Bus Ind 27(6):723-731

Eryilmaz S (2010) Mean residual and mean past lifetime of multi-state systems with identical components. IEEE Trans Reliab 59(4):644-649

He QM (2014) Fundamental of matrix analytic methods. Springer Science+Business Media, New York

Laggounea R, Chateauneuf A, Aissania D (2010) Preventive maintenance scheduling for a multi-component system with non-negligible replacement time. Int J Syst Sci 41(7):747-761

Li W, Pham H (2005) Reliability modeling of multi-state degraded systems with multi-competing failures and random shocks. IEEE Trans Reliab 54(2):297-303

Lisnianski A, Frenkel I, Ding Y (2010) Multi-state system reliability analysis and optimization for engineers and industrial managers. Springer-Verlag, London

Liu Z, Ma X, Shenc L, Zhaob Y (2016) Degradation-shock-based reliability models for fault-tolerant systems. Qual Reliab Eng Int 32(3):949-955

Mahfoud H, El Barkany A, El Biyaali A (2016) Preventive maintenance optimization in healthcare domain: status of research and perspective. J Qual Reliab Eng 2016(Article ID 5314312):10

Murchland J (1975) Fundamental concepts and relations for reliability analysis of multistate systems. In: Barlow RE, Fussell JB, Singpurwalla N (eds) Reliability and fault tree analysis: theoretical and applied aspects of system reliability. SIAM, Philadelphia, pp 581-618

Neuts MF (1975) Probability distributions of phase type. In: Liber Amicorum Prof. Emeritus H. Florin. Department of Mathematics, University of Louvain, Belgium, pp 183-206

Neuts MF (1979) A versatile Markovian point process. J Appl Probab 16:764-779

Neuts MF (1981) Matrix geometric solutions in stochastic models. An algorithmic approach. John Hopkins, University Press, Baltimore

Osaki S, Asakura A (1970) A two-unit standby redundant system with repair and preventive maintenance. J Appl Probab 7:641-648

Ruiz-Castro JE (2016a) Markov counting and reward processes for analyzing the performance of a complex system subject to random inspections. Reliab Eng Syst Saf 145:155-168

Ruiz-Castro JE (2016b) Complex multi-state systems modelled through Marked Markovian Arrival Processes. Eur J Oper Res 252(3):852-865

Ruiz-Castro JE, Li Q-L (2011) Algorithm for a general discrete $k$-out-of- $n$ : $G$ system subject to several types of failure with an indefinite number of repairpersons. Eur J Oper Res 211:97-111

Shatnawi O (2016) An integrated framework for developing discrete-time modelling in software reliability engineering. Qual Reliab Eng Int 32(8):2925-2943

Warrington L, Jones JA (2003) Representing complex systems within discrete event simulation for reliability assessment. In: Proceedings Annual Reliability and Maintainability Symposium, pp 487-492

Yin H, Yang X, Peng R (2015) Generalized Accelerated Failure Time Frailty Model for Systems Subject to Imperfect Preventive Maintenance. Math Probl Eng 2015(Article ID 908742):8 


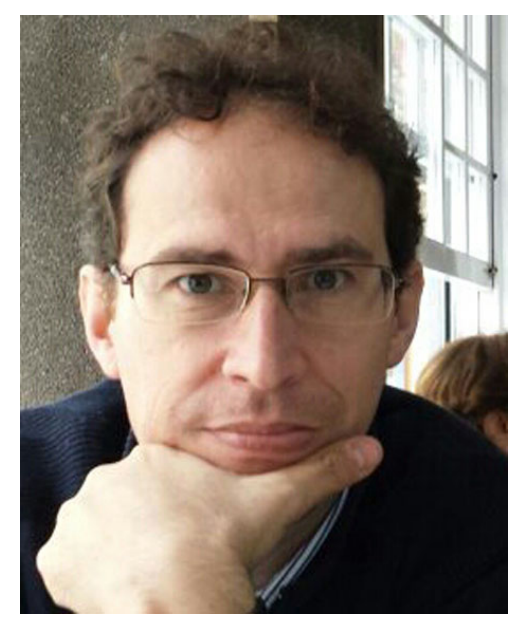

Juan Eloy Ruiz-Castro is a Professor in the Department of Statistics and Operational Research of the University of Granada (Spain). He has worked, from 1993, principally in survival and reliability analysis by considering Markovian and semi-Markovian models. One interest of his research is the applicability of the theoretic models built. In medicine, he has applied these models to the behaviour of breast cancer with several treatments as well as in reliability for analysing repairable systems. As a consequence of this research, he has publications in a great number of high-impact scientific journals and he belongs to the editorial board of several journals with recognized prestigious. Now he is interested in the incorporation of the phase type, matrix exponential distributions and Markovian arrival processes in these fields. 